Supporting Information for:

\title{
Highly Chemo-, Regio-, and Stereoselective [3+2]-Cyclization of Activated and Deactivated Allenes with Alkenyl Fischer Carbene Complexes: A Straightforward Access to
} Alkylidenecyclopentanone Derivatives José Barluenga, * Rubén Vicente, Luis A. López, and Miguel Tomás Instituto Universitario de Química Organometálica “Enrique Moles”, Unidad Asociada al CSIC, Universidad de Oviedo, Julián Clavería 8, 33071 Oviedo, Spain

barluenga@uniovi.es

General Methods: ${ }^{1} \mathrm{H}$ NMR and ${ }^{13} \mathrm{C}$ NMR spectra were recorded at room temperature in $\mathrm{CDCl}_{3}$, on a Bruker AC-300 (300 and 75.5 MHz) and Bruker AMX-400 (400 and $100 \mathrm{MHz})$ spectrometers. Chemical shifts are given in ppm relative to TMS $\left({ }^{1} \mathrm{H}, 0.0\right.$ ppm) or $\mathrm{CDCl}_{3}\left({ }^{13} \mathrm{C}, 77.0 \mathrm{ppm}\right)$. Carbon multiplicities were assigned by DEPT techniques. IR spectra were obtained in a Mattson 3000 FTIR spectrometer. Elemental analyses were carried out on a Perkin-Elmer 2400 and Carlo Erba 1108 microanalyzers. TLC was performed on aluminum-backed plates with silica gel 60 with $\mathrm{F}_{254}$ indicator. All reactions involving organometallic species were carried out under nitrogen using standard Schlenck techniques. $\mathrm{CH}_{2} \mathrm{Cl}_{2}$ was distilled from $\mathrm{CaH}_{2}$. Flash column chromatography was carried out on silica gel (230-240 mesh). Fischer carbene 
complexes 1, allenes $\mathbf{2}, \mathbf{3}$, and $\left[\mathrm{Rh}(\right.$ naphthalene) $(\mathrm{cod})]\left[\mathrm{SbF}_{6}\right]$ were prepared according to literature methods. All other reagents and solvents used in this work were of the best commercial grade available and used without further purification.

General Procedure for the Rh(I)-Catalyzed [3+2]-Cyclization Reaction of Alkenyl Fischer Carbene Complexes 1 and Electron-rich Allenes 2a-c and 2e: Synthesis of Cyclopentene Derivatives 4 and 6.

To a solution of the carbene complex 1 (1 equiv) and the corresponding allene ( 2 equiv for allenes $\mathbf{2 a}$ and $\mathbf{2 e} ; 1.2$ equiv for allenes $\mathbf{2 b}, \mathbf{2 c}$ and $\mathbf{2 d}$ ) in $\mathrm{CH}_{2} \mathrm{Cl}_{2}$ under a $\mathrm{CO}$ atmosphere ( 1 bar) was added the corresponding $\mathrm{Rh}(\mathrm{I})$-catalyst $(0.1$ equiv, $10 \%)$. The mixture was stirred at room temperature until dissaperance of the starting carbene complex (checked by TLC; 0.5-18 h). The solvent was distilled under reduced pressure and the residue was dissolved in a mixture of diethylether and $\mathrm{CH}_{2} \mathrm{Cl}_{2}$ (10:1) and filtered through Celite. The solvents were removed in vacuo and the residue was purified by flash chromatography $\left(\mathrm{SiO}_{2}\right.$, mixtures of hexane and ethyl acetate).

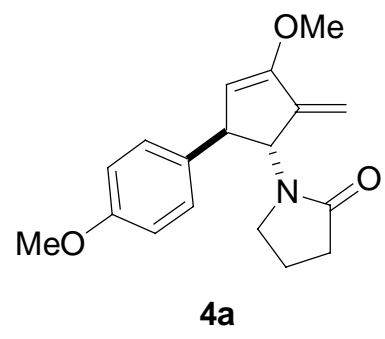

Compound 4a. The general procedure was followed using complex 1a (184 mg, 0.5 $\mathrm{mmol})$, allene $2 \mathbf{a}(123 \mathrm{mg}, 1 \mathrm{mmol})$ and $[\mathrm{Rh}(\mathrm{cod}) \mathrm{Cl}]_{2}(24.6 \mathrm{mg}, 0.05 \mathrm{mmol})$ in $\mathrm{CH}_{2} \mathrm{Cl}_{2}$ (10 mL). Final chromatographic purification using a 2:1 mixture of hexane:ethyl acetate as eluent afforded 4a $(114 \mathrm{mg}, 76 \%) .{ }^{1} \mathrm{H}-\mathrm{NMR}\left(\mathrm{CDCl}_{3}\right): 1.98-2.12(\mathrm{~m}, 2 \mathrm{H}), 2.43(\mathrm{t}, J=$ $7.5 \mathrm{~Hz}, 2 \mathrm{H}), 3.33(\mathrm{t}, J=7.0 \mathrm{~Hz}, 2 \mathrm{H}), 3.70-3.72(\mathrm{~m}, 1 \mathrm{H}), 3.75(\mathrm{~s}, 3 \mathrm{H}), 3.77$ (s, 3H), 4.77 (br s, 1H), 5.04 (br s, 1H), 5.07-5.09 (m, 1H), $5.25(\mathrm{~d}, J=2.5 \mathrm{~Hz}, 1 \mathrm{H}), 6.83(\mathrm{~d}, J=8.8$ 
$\mathrm{Hz}, 2 \mathrm{H}), 7.11(\mathrm{~d}, J=8.8 \mathrm{~Hz}, 2 \mathrm{H}) ;{ }^{13} \mathrm{C}-\mathrm{NMR}\left(\mathrm{CDCl}_{3}\right): 18.1\left(\mathrm{CH}_{2}\right), 31.2\left(\mathrm{CH}_{2}\right), 42.8$ $\left(\mathrm{CH}_{2}\right), 48.2(\mathrm{CH}), 55.2\left(\mathrm{CH}_{3}\right), 56.4\left(\mathrm{CH}_{3}\right), 60.2(\mathrm{CH}), 104.0\left(\mathrm{CH}_{2}\right), 105.9(\mathrm{CH}), 114.0$ $(\mathrm{CH}), 128.0(\mathrm{CH}), 135.4(\mathrm{C}), 144.2(\mathrm{C}), 157.4(\mathrm{C}), 158.5(\mathrm{C}), 174.9(\mathrm{C}) . \mathrm{IR}\left(\mathrm{CH}_{2} \mathrm{Cl}_{2}\right)$ : v 1679, 1611, 1512, $1421 \mathrm{~cm}^{-1}$. Anal. Calcd for $\mathrm{C}_{18} \mathrm{H}_{21} \mathrm{NO}_{3}: \mathrm{C}, 72.22 ; \mathrm{H}, 7.07 ; \mathrm{N}, 4.68$; Found: C, 72.36; H, 7.02; N, 4.65.

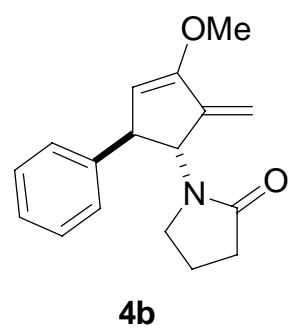

Compound 4b. The general procedure was followed using complex $\mathbf{1 b}$ (169 $\mathrm{mg}, 0.5$ $\mathrm{mmol})$, allene $2 \mathbf{a}(123 \mathrm{mg}, 1 \mathrm{mmol})$ and $[\mathrm{Rh}(\mathrm{cod}) \mathrm{Cl}]_{2}(24.6 \mathrm{mg}, 0.05 \mathrm{mmol})$ in $\mathrm{CH}_{2} \mathrm{Cl}_{2}$ (10 mL). Final chromatographic purification using a 2:1 mixture of hexane:ethyl acetate as eluent afforded $\mathbf{4 b}(105 \mathrm{mg}, 78 \%) .{ }^{1} \mathrm{H}-\mathrm{NMR}\left(\mathrm{CDCl}_{3}\right): 2.01-2.09(\mathrm{~m}, 2 \mathrm{H}), 2.43(\mathrm{t}, J=$ $8.1 \mathrm{~Hz}, 2 \mathrm{H}), 3.35$ (t, $J=7.0 \mathrm{~Hz}, 2 \mathrm{H}), 3.74-3.76(\mathrm{~s}+\mathrm{m}, 4 \mathrm{H}), 4.79$ (br s, 1H), 5.08 (br s, 1H), 5.11-5.14 (m, 1H), $5.27(\mathrm{~d}, J=2.4 \mathrm{~Hz}, 1 \mathrm{H}), 7.19-7.30(\mathrm{~m}, 5 \mathrm{H}) ;{ }^{13} \mathrm{C}-\mathrm{NMR}$ $\left(\mathrm{CDCl}_{3}\right): 18.1\left(\mathrm{CH}_{2}\right), 31.2\left(\mathrm{CH}_{2}\right), 42.8\left(\mathrm{CH}_{2}\right), 49.0(\mathrm{CH}), 56.5\left(\mathrm{CH}_{3}\right), 60.0(\mathrm{CH}), 104.1$ $\left(\mathrm{CH}_{2}\right), 105.7(\mathrm{CH}), 126.8(\mathrm{CH}), 127.0(\mathrm{CH}), 128.6(\mathrm{CH}), 143.3(\mathrm{C}), 144.2(\mathrm{C}), 157.5$ (C), 174.9 (C). IR $\left(\mathrm{CH}_{2} \mathrm{Cl}_{2}\right): v 1682,1422 \mathrm{~cm}^{-1}$. Anal. Calcd for $\mathrm{C}_{17} \mathrm{H}_{19} \mathrm{NO}_{2}: \mathrm{C}, 75.81$; H, 7.11; N, 5.20; Found: C, 75.66; H, 7.13; N, 5.31.

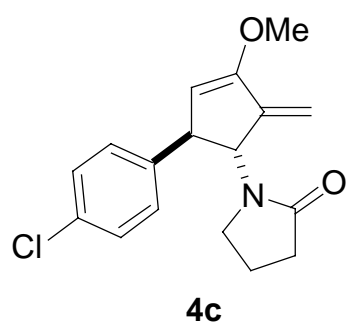

Compound 4c. The general procedure was followed using complex 1c (186 mg, 0.5 $\mathrm{mmol})$, allene $2 \mathbf{a}(123 \mathrm{mg}, 1 \mathrm{mmol})$ and $[\mathrm{Rh}(\mathrm{cod}) \mathrm{Cl}]_{2}(24.6 \mathrm{mg}, 0.05 \mathrm{mmol})$ in $\mathrm{CH}_{2} \mathrm{Cl}_{2}$ 
(10 mL). Final chromatographic purification using a 2:1 mixture of hexane:ethyl acetate as eluent afforded $4 \mathbf{c}(126 \mathrm{mg}, 83 \%) .{ }^{1} \mathrm{H}-\mathrm{NMR}\left(\mathrm{CDCl}_{3}\right): 2.06-2.13(\mathrm{~m}, 2 \mathrm{H}), 2.47(\mathrm{t}, J=$ $8.0 \mathrm{~Hz}, 2 \mathrm{H}), 3.36(\mathrm{t}, \mathrm{J}=7.6 \mathrm{~Hz}, 2 \mathrm{H}), 3.75-3.76(\mathrm{~m}, 1 \mathrm{H}), 3.80(\mathrm{~s}, 3 \mathrm{H}), 4.84(\mathrm{br} \mathrm{s}, 1 \mathrm{H})$, 5.07 (br s, 1H), 5.10-5.12 (m, 1H), $5.32(\mathrm{~d}, J=3.0 \mathrm{~Hz}, 1 \mathrm{H}), 7.18(\mathrm{~d}, J=8.7 \mathrm{~Hz}, 2 \mathrm{H})$, $7.29(\mathrm{~d}, J=8.7 \mathrm{~Hz}, 2 \mathrm{H}) ;{ }^{13} \mathrm{C}-\mathrm{NMR}\left(\mathrm{CDCl}_{3}\right): 18.6\left(\mathrm{CH}_{2}\right), 31.7\left(\mathrm{CH}_{2}\right), 43.2\left(\mathrm{CH}_{2}\right), 49.1$ $(\mathrm{CH}), 57.0\left(\mathrm{CH}_{3}\right), 60.4(\mathrm{CH}), 105.1\left(\mathrm{CH}_{2}\right), 105.5(\mathrm{CH}), 128.9(\mathrm{CH}), 129.2(\mathrm{CH}), 133.0$ (C), $142.4(\mathrm{C}), 144.2(\mathrm{C}), 158.3(\mathrm{C}), 175.5(\mathrm{C}) . \mathrm{IR}\left(\mathrm{CH}_{2} \mathrm{Cl}_{2}\right): v 1680,1612,1491,1422$ $\mathrm{cm}^{-1}$.Anal. Calcd for $\mathrm{C}_{17} \mathrm{H}_{18} \mathrm{ClNO}_{2}: \mathrm{C}, 67.21 ; \mathrm{H}, 5.97 ; \mathrm{N}, 4.61$; Found: $\mathrm{C}, 67.43 ; \mathrm{H}$, $5.83 ; \mathrm{N}, 4.72$.

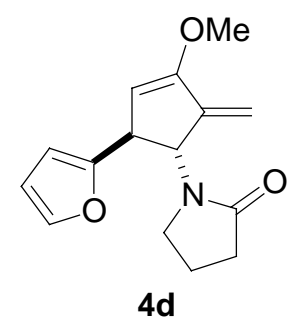

Compound 4d. The general procedure was followed using complex 1d (164 mg, 0.5 $\mathrm{mmol})$, allene $2 \mathbf{a}(123 \mathrm{mg}, 1 \mathrm{mmol})$ and $[\mathrm{Rh}(\mathrm{cod}) \mathrm{Cl}]_{2}(24.6 \mathrm{mg}, 0.05 \mathrm{mmol})$ in $\mathrm{CH}_{2} \mathrm{Cl}_{2}$ (10 mL). Final chromatographic purification using a 2:1 mixture of hexane:ethyl acetate as eluent afforded 4d (104 mg, $80 \%) .{ }^{1} \mathrm{H}-\mathrm{NMR}\left(\mathrm{CDCl}_{3}\right): 1.99-2.09(\mathrm{~m}, 2 \mathrm{H}), 2.45(\mathrm{t}, J=$ 7.7 Hz, 2H), 3.24-3.34 (m, 2H), 3.73 (s, 3H), 3.85-3.87 (m, 1H), 4.79 (br s, 1H), 5.08 (br s, 1H), 5.27 (d, $J=2.5 \mathrm{~Hz}, 1 \mathrm{H}), 5.34-5.36(\mathrm{~m}, 1 \mathrm{H}), 6.17(\mathrm{~d}, J=3.1 \mathrm{~Hz}, 1 \mathrm{H}), 6.27-$ $6.29(\mathrm{~m}, 1 \mathrm{H}), 7.33($ br s, $1 \mathrm{H}) ;{ }^{13} \mathrm{C}-\mathrm{NMR}\left(\mathrm{CDCl}_{3}\right): 18.0\left(\mathrm{CH}_{2}\right), 31.1\left(\mathrm{CH}_{2}\right), 42.3(\mathrm{CH})$, $42.6\left(\mathrm{CH}_{2}\right), 56.4\left(\mathrm{CH}_{3}\right), 56.8(\mathrm{CH}), 102.7(\mathrm{CH}), 104.1\left(\mathrm{CH}_{2}\right), 105.0(\mathrm{CH}), 110.2(\mathrm{CH})$, $141.6(\mathrm{CH}), 143.3(\mathrm{C}), 155.8(\mathrm{C}), 157.4(\mathrm{C}), 175.0(\mathrm{C}) . \mathrm{IR}\left(\mathrm{CH}_{2} \mathrm{Cl}_{2}\right): \vee$ 1682, 1614, $1422 \mathrm{~cm}^{-1}$. Anal. Calcd for $\mathrm{C}_{15} \mathrm{H}_{17} \mathrm{NO}_{3}$ : C, 69.48; H, 6.61; N, 5.40; Found: $\mathrm{C}, 69.43 ; \mathrm{H}$, $6.75 ; \mathrm{N}, 5.44$. 


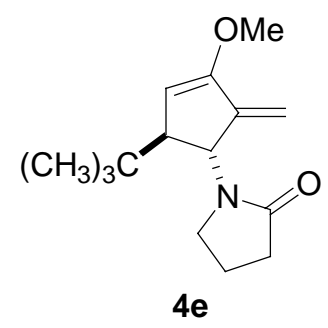

Compound 4e. The general procedure was followed using complex $1 \mathrm{~g}$ (159 mg, 0.5 $\mathrm{mmol})$, allene $\mathbf{2 a}(123 \mathrm{mg}, 1 \mathrm{mmol})$ and $[\mathrm{Rh}(\mathrm{cod}) \mathrm{Cl}]_{2}(24.6 \mathrm{mg}, 0.05 \mathrm{mmol})$ in $\mathrm{CH}_{2} \mathrm{Cl}_{2}$ $(10 \mathrm{~mL})$. Final chromatographic purification using a 2:1 mixture of hexane:ethyl acetate as eluent afforded $4 \mathbf{e}(78 \mathrm{mg}, 63 \%) .{ }^{1} \mathrm{H}-\mathrm{NMR}\left(\mathrm{CDCl}_{3}\right)$ : $0.91(\mathrm{~s}, 9 \mathrm{H}), 1.91-2.06(\mathrm{~m}, 2 \mathrm{H})$, 2.33-2.46 (m, 3H), $3.20(\mathrm{t}, J=7.4 \mathrm{~Hz}, 2 \mathrm{H}), 3.71(\mathrm{~s}, 3 \mathrm{H}), 4.74(\mathrm{br} \mathrm{s}, 1 \mathrm{H}), 4.98(\mathrm{br} \mathrm{s}, 1 \mathrm{H})$, 5.04-5.07 (m, 1H), $5.16(\mathrm{~d}, J=2.4 \mathrm{~Hz}, 1 \mathrm{H}) ;{ }^{13} \mathrm{C}-\mathrm{NMR}\left(\mathrm{CDCl}_{3}\right): 18.0\left(\mathrm{CH}_{2}\right), 26.9\left(\mathrm{CH}_{3}\right)$, $31.3\left(\mathrm{CH}_{2}\right), 33.0(\mathrm{C}), 42.3\left(\mathrm{CH}_{2}\right), 52.7(\mathrm{CH}), 54.0(\mathrm{CH}), 56.2\left(\mathrm{CH}_{3}\right), 103.0\left(\mathrm{CH}_{2}\right), 104.3$ (CH), $145.0(\mathrm{C}), 157.1(\mathrm{C}), 174.2(\mathrm{C}) . \mathrm{IR}\left(\mathrm{CH}_{2} \mathrm{Cl}_{2}\right): v$ 1672, 1613, 1462, 1424, 1270, $1163 \mathrm{~cm}^{-1}$.Anal. Calcd for $\mathrm{C}_{15} \mathrm{H}_{23} \mathrm{NO}_{2}$ : C, 72.25; H, 9.30; N, 5.62; Found: C, 72.18; H, $9.15 ; \mathrm{N}, 5.66$.

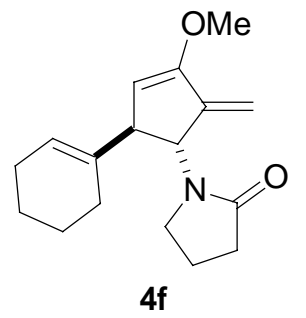

Compound 4f. The general procedure was followed using complex $\mathbf{1 h}(171 \mathrm{mg}, 0.5$ $\mathrm{mmol})$, allene $2 \mathbf{a}(123 \mathrm{mg}, 1 \mathrm{mmol})$ and $[\mathrm{Rh}(\mathrm{cod}) \mathrm{Cl}]_{2}(24.6 \mathrm{mg}, 0.05 \mathrm{mmol})$ in $\mathrm{CH}_{2} \mathrm{Cl}_{2}$ (10 mL). Final chromatographic purification using a 2:1 mixture of hexane:ethyl acetate as eluent afforded $\mathbf{4 f}(107 \mathrm{mg}, 78 \%) .{ }^{1} \mathrm{H}-\mathrm{NMR}\left(\mathrm{CDCl}_{3}\right): 1.54-1.61(\mathrm{~m}, 4 \mathrm{H}), 1.84-2.04$ (m, 6H), $2.43(\mathrm{t}, J=7.5 \mathrm{~Hz}, 2 \mathrm{H}), 3.02(\mathrm{br} \mathrm{s}, 1 \mathrm{H}), 3.15-3.29(\mathrm{~m}, 2 \mathrm{H}), 3.71(\mathrm{~s}, 3 \mathrm{H}), 4.72$ (br s, 1H), 4.89 (br s, 1H), 4.98-5.01 (m, 1H), 5.17 (d, J = 2.3 Hz, 1H), 5.45 (br s, 1H); ${ }^{13} \mathrm{C}-\mathrm{NMR}\left(\mathrm{CDCl}_{3}\right): 18.0\left(\mathrm{CH}_{2}\right), 22.4\left(\mathrm{CH}_{2}\right), 22.8\left(\mathrm{CH}_{2}\right), 25.1\left(\mathrm{CH}_{2}\right), 26.3\left(\mathrm{CH}_{2}\right), 31.3$ 
$\left(\mathrm{CH}_{2}\right), 42.5\left(\mathrm{CH}_{2}\right), 50.4(\mathrm{CH}), 56.3\left(\mathrm{CH}_{3}\right), 56.6(\mathrm{CH}), 103.3\left(\mathrm{CH}_{2}\right), 105.1(\mathrm{CH}), 121.8$ (CH), $138.6(\mathrm{C}), 144.5(\mathrm{C}), 157.0(\mathrm{C}), 174.8(\mathrm{C}) . \mathrm{IR}\left(\mathrm{CH}_{2} \mathrm{Cl}_{2}\right): \vee 1678,1435,1102 \mathrm{~cm}^{-}$ ${ }^{1}$.Anal. Calcd for $\mathrm{C}_{17} \mathrm{H}_{23} \mathrm{NO}_{2}: \mathrm{C}, 74.69 ; \mathrm{H}, 8.48 ; \mathrm{N}, 5.12$; Found: $\mathrm{C}, 74.72 ; \mathrm{H}, 8.43 ; \mathrm{N}$, 5.16.

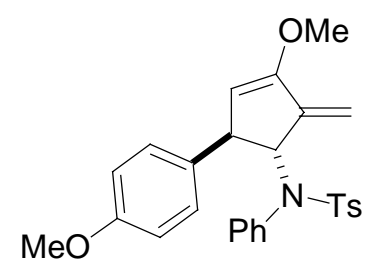

$4 g$

Compound 4g. The general procedure was followed using complex 1a (184 mg, 0.5 $\mathrm{mmol})$, allene $\mathbf{2 b}(171 \mathrm{mg}, 0.6 \mathrm{mmol})$ and $[\mathrm{Rh}(\operatorname{cod}) \mathrm{Cl}]_{2}(24.6 \mathrm{mg}, 0.05 \mathrm{mmol})$ in $\mathrm{CH}_{2} \mathrm{Cl}_{2}(10 \mathrm{~mL})$. Final chromatographic purification using a 5:1 mixture of hexane:ethyl acetate as eluent afforded 4g (226 mg, 98\%). ${ }^{1} \mathrm{H}-\mathrm{NMR}\left(\mathrm{CDCl}_{3}\right): 2.38$ (s, 3H), $3.60(\mathrm{~s}, 3 \mathrm{H}), 3.63-3.65(\mathrm{~m}, 1 \mathrm{H}), 3.84(\mathrm{~s}, 3 \mathrm{H}), 4.70$ (br s, 1H), 5.26-5.29 (m, 1H), $5.35(\mathrm{br} \mathrm{s}, 1 \mathrm{H}), 5.40(\mathrm{~d}, J=2.5 \mathrm{~Hz}, 1 \mathrm{H}), 6.81(\mathrm{~d}, J=8.8 \mathrm{~Hz}, 2 \mathrm{H}), 7.01-7.06(\mathrm{~m}, 4 \mathrm{H})$, 7.14-7.16 (m, 2H), 7.28-7.35 (m, 5H); ${ }^{13} \mathrm{C}-\mathrm{NMR}\left(\mathrm{CDCl}_{3}\right): 21.4\left(\mathrm{CH}_{3}\right), 48.6(\mathrm{CH}), 55.2$ $\left(\mathrm{CH}_{3}\right), 56.1\left(\mathrm{CH}_{3}\right), 69.4(\mathrm{CH}), 105.7\left(\mathrm{CH}_{2}\right), 113.9(\mathrm{CH}), 127.2(\mathrm{CH}), 128.3(\mathrm{CH}), 128.5$ (CH), $128.9(\mathrm{CH}), 129.1(\mathrm{CH}), 131.8(\mathrm{CH}), 135.5(\mathrm{C}), 136.8(\mathrm{C}), 138.3(\mathrm{C}), 142.7(\mathrm{C})$, 145.9 (C), 156.7 (C), 158.5 (C). Anal. Calcd for $\mathrm{C}_{27} \mathrm{H}_{27} \mathrm{NO}_{4} \mathrm{~S}$ : C, 70.26; H, 5.90; N, 3.03; Found: C, 70.21; H, 6.01; N, 3.12.

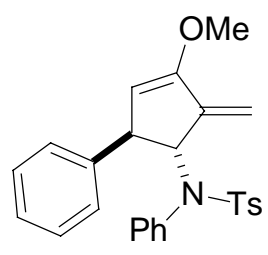

$4 \mathrm{~h}$

Compound 4h. The general procedure was followed using complex $\mathbf{1 b}$ (169 $\mathrm{mg}, 0.5$ $\mathrm{mmol})$, allene $\mathbf{2 b}(171 \mathrm{mg}, 0.6 \mathrm{mmol})$ and $[\mathrm{Rh}(\operatorname{cod}) \mathrm{Cl}]_{2}(24.6 \mathrm{mg}, 0.05 \mathrm{mmol})$ in 
$\mathrm{CH}_{2} \mathrm{Cl}_{2}(10 \mathrm{~mL})$. Final chromatographic purification using a 5:1 mixture of hexane:ethyl acetate as eluent afforded 4 h $(190 \mathrm{mg}, 88 \%) .{ }^{1} \mathrm{H}-\mathrm{NMR}\left(\mathrm{CDCl}_{3}\right): 2.37$ (s, $3 \mathrm{H}), 3.60(\mathrm{~s}, 3 \mathrm{H}), 3.68-3.70(\mathrm{~m}, 1 \mathrm{H}), 4.72(\mathrm{br} \mathrm{s}, 1 \mathrm{H}), 5.33-5.35(\mathrm{~m}, 1 \mathrm{H}), 5.37$ (br s, $1 \mathrm{H})$, $5.42(\mathrm{~d}, J=2.3 \mathrm{~Hz}, 1 \mathrm{H}), 7.03(\mathrm{~d}, J=8.5 \mathrm{~Hz}, 2 \mathrm{H}), 7.10-7.27(\mathrm{~m}, 12 \mathrm{H}) ;{ }^{13} \mathrm{C}-\mathrm{NMR}$ $\left(\mathrm{CDCl}_{3}\right): 21.4\left(\mathrm{CH}_{3}\right), 49.5(\mathrm{CH}), 56.2\left(\mathrm{CH}_{3}\right), 69.2(\mathrm{CH}), 105.6(\mathrm{CH}), 106.0\left(\mathrm{CH}_{2}\right)$, $126.8(\mathrm{CH}), 127.3(\mathrm{CH}), 127.6(\mathrm{CH}), 128.3(\mathrm{CH}), 128.6(\mathrm{CH}), 128.9(\mathrm{CH}), 129.2(\mathrm{CH})$, $131.9(\mathrm{CH}), 136.8(\mathrm{C}), 138.3(\mathrm{C}), 142.8$ (C), 143.6 (C), 145.9 (C), 157.0 (C). IR $\left(\mathrm{CH}_{2} \mathrm{Cl}_{2}\right): v 1614,1422,1162 \mathrm{~cm}^{-1}$. Anal. Calcd for $\mathrm{C}_{26} \mathrm{H}_{25} \mathrm{NO}_{3} \mathrm{~S}: \mathrm{C}, 72.36 ; \mathrm{H}, 5.84 ; \mathrm{N}$, 3.25; Found: C, 72.29; H, 5.91; N, 3.22.

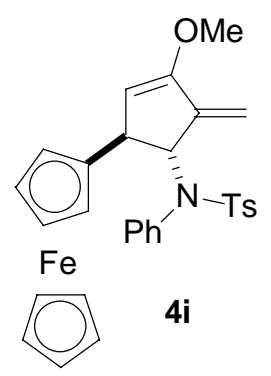

Compound 4i. The general procedure was followed using complex 1e (223 mg, 0.5 $\mathrm{mmol})$, allene $\mathbf{2 b}(171 \mathrm{mg}, 0.6 \mathrm{mmol})$ and $[\mathrm{Rh}(\operatorname{cod}) \mathrm{Cl}]_{2}(24.6 \mathrm{mg}, 0.05 \mathrm{mmol})$ in $\mathrm{CH}_{2} \mathrm{Cl}_{2}$ (10 mL). Final chromatographic purification using a 5:1 mixture of hexane:ethyl acetate as eluent afforded 4i $(267 \mathrm{mg}, 99 \%) .{ }^{1} \mathrm{H}-\mathrm{NMR}\left(\mathrm{CDCl}_{3}\right): 2.41$ (s, 3H), $3.45(\mathrm{t}, J=3.0 \mathrm{~Hz}, 1 \mathrm{H}), 3.64(\mathrm{~s}, 3 \mathrm{H}), 3.93(\mathrm{br} \mathrm{s}, 1 \mathrm{H}), 4.07-4.16$ (several signals, 8H), 4.91 (br s, 1H), 5.13 (br s, 1H), 5.19-5.22 (m, 1H), 5.31 (d, J = 2.4 Hz, 1H), 7.13$7.30(\mathrm{~m}, 7 \mathrm{H}), 7.51(\mathrm{~d}, J=8.2 \mathrm{~Hz}, 2 \mathrm{H}) ;{ }^{13} \mathrm{C}-\mathrm{NMR}\left(\mathrm{CDCl}_{3}\right): 21.5\left(\mathrm{CH}_{3}\right), 43.5(\mathrm{CH}), 56.2$ $\left(\mathrm{CH}_{3}\right), 65.9(\mathrm{CH}), 67.4(\mathrm{CH}), 67.5,67.7(\mathrm{CH}), 68.3(\mathrm{CH}), 91.7(\mathrm{C}), 104.9(\mathrm{CH}), 105.8$ $\left(\mathrm{CH}_{2}\right), 127.6(\mathrm{CH}), 128.3(\mathrm{CH}), 128.8(\mathrm{CH}), 129.3(\mathrm{CH}), 131.9(\mathrm{CH}), 136.8(\mathrm{C}), 138.4$ (C), 143.0 (C), 146.3 (C), 156.5 (C). Anal. Calcd for $\mathrm{C}_{30} \mathrm{H}_{29} \mathrm{FeNO}_{3} \mathrm{~S}$ : C, 66.79; H, 5.42; N, 2.60; Found: C, 66.90; H, 5.46; N, 2.71. 


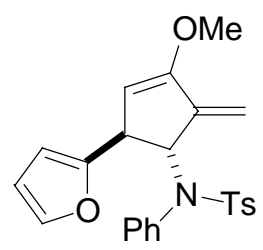

$4 \mathbf{j}$

Compound 4j. The general procedure was followed using complex 1d (164 mg, 0.5 mmol), allene $2 \mathbf{b}(172 \mathrm{mg}, 0.6 \mathrm{mmol})$ and $[\mathrm{Rh}(\mathrm{cod}) \mathrm{Cl}]_{2}(24.6 \mathrm{mg}, 0.05 \mathrm{mmol})$ in $\mathrm{CH}_{2} \mathrm{Cl}_{2}(10 \mathrm{~mL})$. Final chromatographic purification using a 5:1 mixture of hexane:ethyl acetate as eluent afforded $\mathbf{4 j}$ (185 mg, $88 \%)$. ${ }^{1} \mathrm{H}-\mathrm{NMR}\left(\mathrm{CDCl}_{3}\right): 2.41$ (s, 3H), $3.60(\mathrm{~s}, 3 \mathrm{H}), 3.82(\mathrm{dd}, J=4.6$ and $2.4 \mathrm{~Hz}, 1 \mathrm{H}), 4.71$ (br s, 1H), 5.36 (br s, 1H), $5.41(\mathrm{~d}, J=2.5 \mathrm{~Hz}, 1 \mathrm{H}), 5.53-5.56(\mathrm{~m}, 1 \mathrm{H}), 6.05(\mathrm{~d}, J=3.1 \mathrm{~Hz}, 1 \mathrm{H}), 6.30-6.32(\mathrm{~m}, 1 \mathrm{H})$, 7.10-7.27 (m, 8H), $7.48(\mathrm{~d}, J=8.0 \mathrm{~Hz}, 2 \mathrm{H}) ;{ }^{13} \mathrm{C}-\mathrm{NMR}\left(\mathrm{CDCl}_{3}\right): 21.4\left(\mathrm{CH}_{3}\right), 42.8(\mathrm{CH})$, $56.1\left(\mathrm{CH}_{3}\right), 66.0(\mathrm{CH}), 102.1(\mathrm{CH}), 105.7(\mathrm{CH}), 106.0\left(\mathrm{CH}_{2}\right), 110.2(\mathrm{CH}), 127.4(\mathrm{CH})$, $128.5(\mathrm{CH}), 128.9(\mathrm{CH}), 129.2(\mathrm{CH}), 132.3(\mathrm{CH}), 136.4(\mathrm{C}), 138.2(\mathrm{C}), 141.5(\mathrm{CH})$, 142.9 (C), 145.0 (C), 155.7 (C), 156.9 (C). Anal. Calcd for $\mathrm{C}_{24} \mathrm{H}_{23} \mathrm{NO}_{4} \mathrm{~S}: \mathrm{C}, 68.39$; $\mathrm{H}$, 5.50; N, 3.32; Found: C, 68.46; H, 5.33; N, 3.33 .

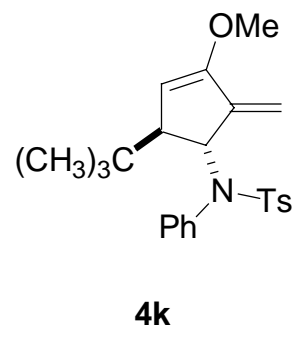

Compound 4k. The general procedure was followed using complex $1 \mathrm{~g}(159 \mathrm{mg}, 0.5$ $\mathrm{mmol})$, allene $2 \mathbf{b}(171 \mathrm{mg}, 0.6 \mathrm{mmol})$ and $[\mathrm{Rh}(\mathrm{cod}) \mathrm{Cl}]_{2}(24.6 \mathrm{mg}, 0.05 \mathrm{mmol})$ in $\mathrm{CH}_{2} \mathrm{Cl}_{2}(10 \mathrm{~mL})$. Final chromatographic purification using a 5:1 mixture of hexane:ethyl acetate as eluent afforded 4k (123 mg, 60\%). ${ }^{1} \mathrm{H}-\mathrm{NMR}\left(\mathrm{CDCl}_{3}\right): 0.93$ (s, 9H), 2.32 (t, $J=2.5 \mathrm{~Hz}, 1 \mathrm{H}), 2.39$ (s, 3H), 3.49 (s, 3H), 4.61 (br s, 1H), 5.10 (br s, 1H), $5.22(\mathrm{br} \mathrm{s}, 1 \mathrm{H}), 5.28-5.32(\mathrm{~m}, 1 \mathrm{H}), 7.11-7.25(\mathrm{~m}, 7 \mathrm{H}), 7.51(\mathrm{~d}, J=8.1 \mathrm{~Hz}, 2 \mathrm{H}) ;{ }^{13} \mathrm{C}-$ 
NMR $\left(\mathrm{CDCl}_{3}\right)$ : $21.5\left(\mathrm{CH}_{3}\right), 27.3\left(\mathrm{CH}_{3}\right), 33.9(\mathrm{C}), 55.0(\mathrm{CH}), 56.1\left(\mathrm{CH}_{3}\right), 60.3(\mathrm{CH})$, $104.1(\mathrm{CH}), 105.9\left(\mathrm{CH}_{2}\right), 127.8(\mathrm{CH}), 128.2(\mathrm{CH}), 128.4(\mathrm{CH}), 129.1(\mathrm{CH}), 132.5$ (CH), 136.3 (C), 138.2 (C), 143.0 (C), 147.3 (C), 157.2 (C). Anal. Calcd for $\mathrm{C}_{24} \mathrm{H}_{29} \mathrm{NO}_{3} \mathrm{~S}$ : C, 70.04; H, 7.10; N, 3.40; Found: C, 70.17; H, 7.02; N, 3.52.

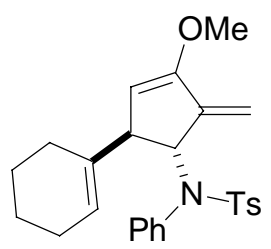

4!

Compound 4l: The general procedure was followed using complex $\mathbf{1 h}(171 \mathrm{mg}, 0.5$ $\mathrm{mmol})$, allene $\mathbf{2 b}(171 \mathrm{mg}, 0.6 \mathrm{mmol})$ and $[\mathrm{Rh}(\mathrm{cod}) \mathrm{Cl}]_{2}(24.6 \mathrm{mg}, 0.05 \mathrm{mmol})$ in $\mathrm{CH}_{2} \mathrm{Cl}_{2}(10 \mathrm{~mL})$. Final chromatographic purification using a 5:1 mixture of hexane:ethyl acetate as eluent afforded 41 (170 mg, 78\%). ${ }^{1} \mathrm{H}-\mathrm{NMR}\left(\mathrm{CDCl}_{3}\right): 1.52-1.66$ (m, 4H), 1.89-2.01 (m, 4H), $2.42(\mathrm{~s}, 3 \mathrm{H}), 3.10-3.11(\mathrm{~m}, 1 \mathrm{H}), 3.54$ (s, 3H), 4.52 (br s, 1H), 5.21-5.23 (m, 2H), $5.28(\mathrm{~d}, J=2.6 \mathrm{~Hz}, 1 \mathrm{H}), 5.36$ (br s, 1H), 7.10-7.28 (m, 7H), $7.62(\mathrm{~d}, J=8.0 \mathrm{~Hz}, 2 \mathrm{H}) ;{ }^{13} \mathrm{C}-\mathrm{NMR}\left(\mathrm{CDCl}_{3}\right): 21.4\left(\mathrm{CH}_{3}\right), 22.4\left(\mathrm{CH}_{2}\right), 22.8\left(\mathrm{CH}_{2}\right), 25.2$ $\left(\mathrm{CH}_{2}\right), 25.3\left(\mathrm{CH}_{2}\right), 51.3(\mathrm{CH}), 56.0\left(\mathrm{CH}_{3}\right), 64.1(\mathrm{CH}), 104.9(\mathrm{CH}), 105.3\left(\mathrm{CH}_{2}\right), 123.4$ $(\mathrm{CH}), 127.6(\mathrm{CH}), 128.2(\mathrm{CH}), 128.7(\mathrm{CH}), 129.2(\mathrm{CH}), 132.3(\mathrm{CH}), 136.5(\mathrm{C}), 138.0$ (C), 138.6 (C), 143.0 (C), 16.2 (C), 156.5 (C). Anal. Calcd for $\mathrm{C}_{26} \mathrm{H}_{29} \mathrm{NO}_{3} \mathrm{~S}: \mathrm{C}, 71.69$; H, 6.71; N, 3.22; Found: C, 71.54; H, 6.73; N, 3.25.

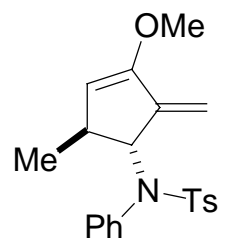

$4 \mathrm{~m}$

Compound 4m. The general procedure was followed using complex 1f (138 mg, 0.5 $\mathrm{mmol})$, allene $2 \mathbf{b}(171 \mathrm{mg}, 0.6 \mathrm{mmol})$ and $[\mathrm{Rh}(\operatorname{cod}) \mathrm{Cl}]_{2}(24.6 \mathrm{mg}, 0.05 \mathrm{mmol})$ in 
$\mathrm{CH}_{2} \mathrm{Cl}_{2}(10 \mathrm{~mL})$. Final chromatographic purification using a 5:1 mixture of hexane:ethyl acetate as eluent afforded $4 \mathrm{~m}(87 \mathrm{mg}, 47 \%) .{ }^{1} \mathrm{H}-\mathrm{NMR}\left(\mathrm{CDCl}_{3}\right): 1.11$ (d, $J$ $=6.8 \mathrm{~Hz}, 3 \mathrm{H}), 2.45(\mathrm{~s}, 3 \mathrm{H}), 2.61-2.65(\mathrm{~m}, 1 \mathrm{H}), 3.53(\mathrm{~s}, 3 \mathrm{H}), 4.63$ (br s, 1H), 4.93-4.94 (m, 1H), 4.97 (br s, 1H), $5.21(\mathrm{~d}, J=2.3 \mathrm{~Hz}, 1 \mathrm{H}), 7.11(\mathrm{~d}, J=8.1 \mathrm{~Hz}, 2 \mathrm{H}), 7.27-7.29$ $(\mathrm{m}, 5 \mathrm{H}), 7.67(\mathrm{~d}, J=8.1 \mathrm{~Hz}, 2 \mathrm{H}) ;{ }^{13} \mathrm{C}-\mathrm{NMR}\left(\mathrm{CDCl}_{3}\right): 19.9\left(\mathrm{CH}_{3}\right), 21.5\left(\mathrm{CH}_{3}\right), 38.7$ $(\mathrm{CH}), 56.0\left(\mathrm{CH}_{3}\right), 67.5(\mathrm{CH}), 105.1\left(\mathrm{CH}_{2}\right), 107.1(\mathrm{CH}), 127.6(\mathrm{CH}), 128.3(\mathrm{CH}), 128.7$ $(\mathrm{CH}), 129.4(\mathrm{CH}), 132.2(\mathrm{CH}), 136.7(\mathrm{C}), 138.6(\mathrm{C}), 143.2(\mathrm{C}), 145.8(\mathrm{C}), 155.9(\mathrm{C})$. Anal. Calcd for $\mathrm{C}_{21} \mathrm{H}_{23} \mathrm{NO}_{3} \mathrm{~S}: \mathrm{C}, 68.27 ; \mathrm{H}, 6.27 ; \mathrm{N}, 3.79$; Found: $\mathrm{C}, 68.39 ; \mathrm{H}, 6.21 ; \mathrm{N}$, 3.83 .

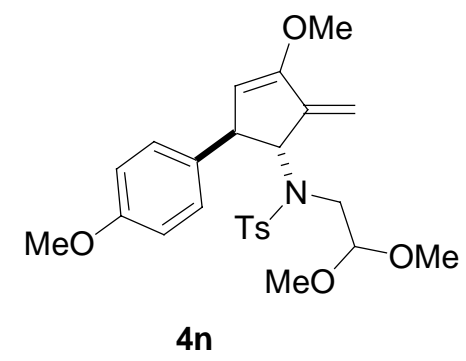

Compound 4n. The general procedure was followed using complex 1a (184 mg, 0.5 $\mathrm{mmol})$, allene $2 \mathrm{c}(178 \mathrm{mg}, 0.6 \mathrm{mmol})$ and $[\mathrm{Rh}(\mathrm{cod}) \mathrm{Cl}]_{2}(24.6 \mathrm{mg}, 0.05 \mathrm{mmol})$ in $\mathrm{CH}_{2} \mathrm{Cl}_{2}(10 \mathrm{~mL})$. Final chromatographic purification using a 3:1 mixture of hexane:ethyl acetate as eluent afforded 4n $(232 \mathrm{mg}, 98 \%) .{ }^{1} \mathrm{H}-\mathrm{NMR}\left(\mathrm{CDCl}_{3}\right): 2.36$ (s, 3H), $3.07(\mathrm{dd}, \mathrm{J}=15.2$ and $7.2 \mathrm{~Hz}, 1 \mathrm{H}), 3.41(\mathrm{~s}, 3 \mathrm{H}), 3.42(\mathrm{~s}, 3 \mathrm{H}), 3.37(\mathrm{dd}, \mathrm{J}=15.2$ and $3.6 \mathrm{~Hz}, 1 \mathrm{H}), 3.68(\mathrm{~s}, 3 \mathrm{H}), 3.79(\mathrm{~s}, 3 \mathrm{H}), 4.04(\mathrm{t}, \mathrm{J}=2.8 \mathrm{~Hz}, 1 \mathrm{H}), 4.56(\mathrm{br} \mathrm{s}, 1 \mathrm{H})$, 4.67-4.71 (m, 2H), $4.88(\mathrm{t}, \mathrm{J}=1.8 \mathrm{~Hz}, 1 \mathrm{H}), 5.15(\mathrm{~d}, \mathrm{~J}=2.4 \mathrm{~Hz}, 1 \mathrm{H}), 6.74(\mathrm{~d}, \mathrm{~J}=8.8 \mathrm{~Hz}$, 2H), $6.98(\mathrm{~d}, \mathrm{~J}=8.4 \mathrm{~Hz}, 2 \mathrm{H}), 7.08(\mathrm{~d}, \mathrm{~J}=8.4 \mathrm{~Hz}, 2 \mathrm{H}), 7.48(\mathrm{~d}, \mathrm{~J}=8.8 \mathrm{~Hz}, 2 \mathrm{H}) ;{ }^{13} \mathrm{C}-$ $\operatorname{NMR}\left(\mathrm{CDCl}_{3}\right): 21.2\left(\mathrm{CH}_{3}\right), 47.3\left(\mathrm{CH}_{2}\right), 48.9(\mathrm{CH}), 54.5\left(\mathrm{CH}_{3}\right), 55.0\left(\mathrm{CH}_{3}\right), 55.3\left(\mathrm{CH}_{3}\right)$, $56.1\left(\mathrm{CH}_{3}\right), 68.0(\mathrm{CH}), 104.0(\mathrm{CH}), 104.7\left(\mathrm{CH}_{2}\right), 106.3(\mathrm{CH}), 113.6(\mathrm{CH}), 127.0(\mathrm{CH})$, $128.3(\mathrm{CH}), 129.2(\mathrm{CH}), 135.7(\mathrm{C}), 137.5(\mathrm{C}), 142.9(\mathrm{C}), 144.2(\mathrm{C}), 156.6(\mathrm{C}), 158.3$ 
(C). Anal. Calcd for $\mathrm{C}_{25} \mathrm{H}_{31} \mathrm{NO}_{6} \mathrm{~S}$ : C, 63.40; H, 6.60; N, 2.96; Found: C, 63.56; H, 6.54;

N, 2.93.

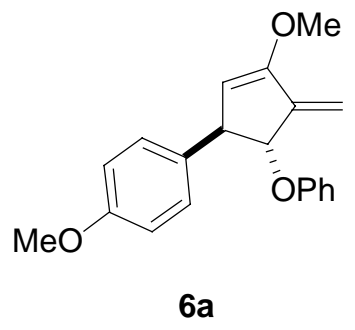

Compound 6a. The general procedure was followed using complex 1a (184 mg, 0.5 $\mathrm{mmol})$, allene $2 \mathrm{e}(132 \mathrm{mg}, 1 \mathrm{mmol})$ and $\left[\mathrm{Rh}(\mathrm{CO})_{2} \mathrm{Cl}\right]_{2}(19.4 \mathrm{mg}, 0.05 \mathrm{mmol})$ in $\mathrm{CH}_{2} \mathrm{Cl}_{2}$ (10 mL). Final chromatographic purification using a 10:1 mixture of hexane:ethyl acetate as eluent afforded 6a (97 mg, 63\%). ${ }^{1} \mathrm{H}-\mathrm{NMR}\left(\mathrm{CDCl}_{3}\right): 3.80(\mathrm{~s}, 3 \mathrm{H}), 3,84(\mathrm{~s}$, $3 \mathrm{H}), 3.90(\mathrm{t}, J=2.3 \mathrm{~Hz}, 1 \mathrm{H}) ; 5.05(\mathrm{~m}, 2 \mathrm{H}), 5.20(\mathrm{br} \mathrm{s}, 1 \mathrm{H}), 5.44(\mathrm{~d}, J=1.6 \mathrm{~Hz}, 1 \mathrm{H})$, 6.80-6.94 (m, 5H), 7.10-7.24 (m, 4H); ${ }^{13} \mathrm{C}-\mathrm{NMR}\left(\mathrm{CDCl}_{3}\right): 51.9(\mathrm{CH}), 55.2\left(\mathrm{CH}_{3}\right), 56.5$ $\left(\mathrm{CH}_{3}\right), 83.9(\mathrm{CH}), 106.0(\mathrm{CH}), 106.9\left(\mathrm{CH}_{2}\right), 114.0(\mathrm{CH}), 116.0(\mathrm{CH}), 121.0(\mathrm{CH}), 128.5$ $(\mathrm{CH}), 129.3(\mathrm{CH}), 135.5(\mathrm{C}), 146.4(\mathrm{C}), 156.7(\mathrm{C}), 157.9(\mathrm{C}), 158.5(\mathrm{C}) . \mathrm{IR}\left(\mathrm{CH}_{2} \mathrm{Cl}_{2}\right)$ : v 1614, 1442, $1164 \mathrm{~cm}^{-1}$. Anal. Calcd for $\mathrm{C}_{20} \mathrm{H}_{20} \mathrm{O}_{3}$ : C, 77.90; H, 6.54; Found: C, 77.95; H, 6.66.

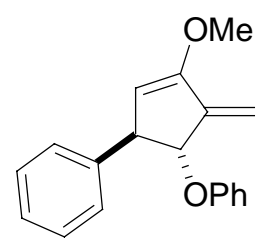

6b

Compound 6b. The general procedure was followed using complex $\mathbf{1 b}$ (169 $\mathrm{mg}, 0.5$ $\mathrm{mmol})$, allene $2 \mathbf{e}(132 \mathrm{mg}, 1 \mathrm{mmol})$ and $\left[\mathrm{Rh}(\mathrm{CO})_{2} \mathrm{Cl}\right]_{2}(19.4 \mathrm{mg}, 0.05 \mathrm{mmol})$ in $\mathrm{CH}_{2} \mathrm{Cl}_{2}$ (10 mL). Final chromatographic purification using a 10:1 mixture of hexane:ethyl acetate as eluent afforded $\mathbf{6 b}(82 \mathrm{mg}, 59 \%) .{ }^{1} \mathrm{H}-\mathrm{NMR}\left(\mathrm{CDCl}_{3}\right): 3.78(\mathrm{~s}, 3 \mathrm{H}), 3.92$ (br s, 1H), 5.06 (br s, 2H), 5.19 (br s, 1H), 5.42 (br s, 1H), 6.81 (d, J= 7.9 Hz, 2H), 6.93 (t, $J$ 
$=7.3 \mathrm{~Hz}, 1 \mathrm{H}), 7.18-7.36(\mathrm{~m}, 7 \mathrm{H}) ;{ }^{13} \mathrm{C}-\mathrm{NMR}\left(\mathrm{CDCl}_{3}\right): 52.7(\mathrm{CH}), 56.5\left(\mathrm{CH}_{3}\right), 83.7$ $(\mathrm{CH}), 105.7(\mathrm{CH}), 107.0\left(\mathrm{CH}_{2}\right), 116.0(\mathrm{CH}), 121.0(\mathrm{CH}), 126.9(\mathrm{CH}), 127.6(\mathrm{CH})$, $128.6(\mathrm{CH}), 129.4(\mathrm{CH}), 143.4(\mathrm{C}), 146.4(\mathrm{C}), 156.9(\mathrm{C}), 157.9(\mathrm{C}) . \mathrm{IR}\left(\mathrm{CH}_{2} \mathrm{Cl}_{2}\right): v$ 1600, 1500, $1164 \mathrm{~cm}^{-1}$. Anal. Calcd for $\mathrm{C}_{19} \mathrm{H}_{18} \mathrm{O}_{2}$ : C, 81.99; H, 6.52; Found: C, 81.84; H, 6.57 .

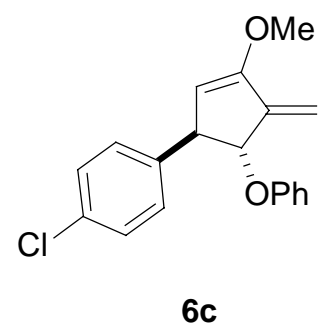

Compound 6c. The general procedure was followed using complex 1c (186 mg, 0.5 $\mathrm{mmol})$, allene $2 \mathbf{e}(132 \mathrm{mg}, 1 \mathrm{mmol})$ and $\left[\mathrm{Rh}(\mathrm{CO})_{2} \mathrm{Cl}\right]_{2}(19.4 \mathrm{mg}, 0.05 \mathrm{mmol})$ in $\mathrm{CH}_{2} \mathrm{Cl}_{2}$ $(10 \mathrm{~mL})$. Final chromatographic purification using a 10:1 mixture of hexane:ethyl acetate as eluent afforded $\mathbf{6 c}(83 \mathrm{mg}, 53 \%) .{ }^{1} \mathrm{H}-\mathrm{NMR}\left(\mathrm{CDCl}_{3}\right): 3.83(\mathrm{~s}, 3 \mathrm{H}), 3.95(\mathrm{t}, J=$ $2.3 \mathrm{~Hz}, 1 \mathrm{H}), 5.05-5.06(\mathrm{~m}, 2 \mathrm{H}), 5.24(\mathrm{br} \mathrm{s}, 1 \mathrm{H}), 5.47$ (d, $J=1.5 \mathrm{~Hz}, 1 \mathrm{H}), 6.85$ (d, $J=$ $7.8 \mathrm{~Hz}, 2 \mathrm{H}), 6.99(\mathrm{t}, J=7.3 \mathrm{~Hz}, 1 \mathrm{H}), 7.16-7.27(\mathrm{~m}, 6 \mathrm{H}) ;{ }^{13} \mathrm{C}-\mathrm{NMR}\left(\mathrm{CDCl}_{3}\right): 52.1(\mathrm{CH})$, 56.6 $\left(\mathrm{CH}_{3}\right), 83.7(\mathrm{CH}), 105.0(\mathrm{CH}), 107.3\left(\mathrm{CH}_{2}\right), 116.0(\mathrm{CH}), 121.2(\mathrm{CH}), 128.8(\mathrm{CH})$, $128.9(\mathrm{CH}), 129.4(\mathrm{CH}), 132.6(\mathrm{C}), 142.0(\mathrm{C}), 146.0(\mathrm{C}), 157.2(\mathrm{C}), 157.8(\mathrm{C})$. Anal. Calcd for $\mathrm{C}_{19} \mathrm{H}_{17} \mathrm{ClO}_{2}$ : C, 72.96; H, 5.48; Found: C, 73.02; H, 5.51.

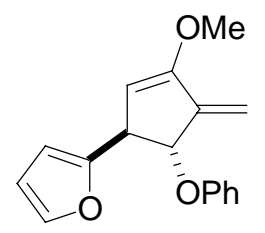

$6 d$

Compound 6d. The general procedure was followed using complex 1d (164 mg, 0.5 $\mathrm{mmol})$, allene $2 \mathrm{e}(132 \mathrm{mg}, 1 \mathrm{mmol})$ and $\left[\mathrm{Rh}(\mathrm{CO})_{2} \mathrm{Cl}\right]_{2}(19.4 \mathrm{mg}, 0.05 \mathrm{mmol})$ in $\mathrm{CH}_{2} \mathrm{Cl}_{2}$ (10 mL). Final chromatographic purification using a 10:1 mixture of hexane:ethyl 
acetate as eluent afforded $\mathbf{6 d}(80 \mathrm{mg}, 60 \%) .{ }^{1} \mathrm{H}-\mathrm{NMR}\left(\mathrm{CDCl}_{3}\right): 3.78(\mathrm{~s}, 3 \mathrm{H}), 4.04$ (br s, 1H), 5.01 (br s, 1H), 5.18 (br s, 1H), 5.22 (br s, 1H), 5.41 (br s, 1H), 6.08 (d, J= 3.3 Hz, 1H), 6.31-6.33 (m, 1H), 6.93-6.98 (m, 3H), $7.26(\mathrm{t}, J=7.1 \mathrm{~Hz}, 2 \mathrm{H}), 7.38(\mathrm{~d}, J=1.0 \mathrm{~Hz}$, $1 \mathrm{H}) ;{ }^{13} \mathrm{C}-\mathrm{NMR}\left(\mathrm{CDCl}_{3}\right): 46.1(\mathrm{CH}), 56.6\left(\mathrm{CH}_{3}\right), 80.8(\mathrm{CH}), 102.0(\mathrm{CH}), 105.8(\mathrm{CH})$, $107.5\left(\mathrm{CH}_{2}\right), 110.3(\mathrm{CH}), 115.9(\mathrm{CH}), 121.2(\mathrm{CH}), 129.4(\mathrm{CH}), 141.8(\mathrm{CH}), 145.5(\mathrm{C})$, 155.8 (C), $157.3(\mathrm{C}), 157.9$ (C). IR $\left(\mathrm{CH}_{2} \mathrm{Cl}_{2}\right): v 1614,1422,1164 \mathrm{~cm}^{-1}$.Anal. Calcd for $\mathrm{C}_{17} \mathrm{H}_{16} \mathrm{O}_{3}$ : C, 76.10; H, 6.01; Found: C, 76.04; H, 5.96.

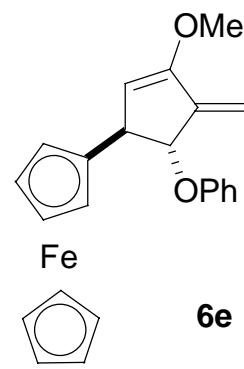

Compound 6e. The general procedure was followed using complex 1e (223 mg, 0.5 $\mathrm{mmol})$, allene $2 \mathbf{e}(132 \mathrm{mg}, 1 \mathrm{mmol})$ and $\left[\mathrm{Rh}(\mathrm{CO})_{2} \mathrm{Cl}\right]_{2}(19.4 \mathrm{mg}, 0.05 \mathrm{mmol})$ in $\mathrm{CH}_{2} \mathrm{Cl}_{2}$ (10 mL). Final chromatographic purification using a 10:1 mixture of hexane:ethyl acetate as eluent afforded 6e (131 mg, 68\%). ${ }^{1} \mathrm{H}-\mathrm{NMR}\left(\mathrm{CDCl}_{3}\right): 3.72$ (br s, $\left.1 \mathrm{H}\right), 3.82$ (s, 3H), 4.00 (br s, 1H), 4.08-4.13 (s+m, 8H), 5.01 (br s, 1H), 5.08 (br s, 1H), 5.16 (br s, $1 \mathrm{H}), 5.31(\mathrm{~d}, J=1.6 \mathrm{~Hz}, 1 \mathrm{H}), 6.95-6.99(\mathrm{~m}, 3 \mathrm{H}), 7.26-7.31(\mathrm{~m}, 2 \mathrm{H}) ;{ }^{13} \mathrm{C}-\mathrm{NMR}$ $\left(\mathrm{CDCl}_{3}\right): 46.9(\mathrm{CH}), 56.9\left(\mathrm{CH}_{3}\right), 67.0(\mathrm{CH}), 68.0(\mathrm{CH}), 68.1(\mathrm{CH}), 68.2(\mathrm{CH}), 68.9$ $(\mathrm{CH}), 84.0(\mathrm{CH}), 91.2(\mathrm{C}), 105.0(\mathrm{CH}), 106.8\left(\mathrm{CH}_{2}\right), 116.7(\mathrm{CH}), 121.6(\mathrm{CH}), 129.9$ $(\mathrm{CH}), 146.9(\mathrm{C}), 156.8(\mathrm{C}), 158.6(\mathrm{C}) . \mathrm{IR}\left(\mathrm{CH}_{2} \mathrm{Cl}_{2}\right): v 1614,1494,1421 \mathrm{~cm}^{-1}$. Anal. Calcd for $\mathrm{C}_{23} \mathrm{H}_{22} \mathrm{FeO}_{2}$ : C, 71.52; H, 5.74; Found: C, 71.60; H, 5.59. 


\section{General Procedure for the Rh(I)-Catalyzed [3+2]-Cyclization Reaction of Alkenyl}

Fischer Carbene Complexes 1 and Electron-Poor Allenes 3a-h: Synthesis of Cyclopentene Derivatives 7-10.

To a solution of the carbene complex 1 ( 1 equiv) and the corresponding allene (2 equiv for allenes 3c-e; 3 equiv for allenes $\mathbf{3 a - b}$ and $\mathbf{3 f}-\mathbf{h})$ in $\mathrm{CH}_{2} \mathrm{Cl}_{2}$ under a $\mathrm{CO}$ atmosphere (1 bar) was added $[\mathrm{Rh}($ naphthalene $)(\mathrm{cod})]\left[\mathrm{SbF}_{6}\right](0.1$ equiv, $10 \%)$. The mixture was stirred at room temperature until dissaperance of the starting carbene complex (checked by TLC; 4-18 h). The solvent was distilled under reduced pressure and the residue was dissolved in a mixture of diethylether and $\mathrm{CH}_{2} \mathrm{Cl}_{2}$ (10:1) and filtered through Celite. The solvent was removed in vacuo and the residue was purified by flash chromatography $\left(\mathrm{SiO}_{2}\right.$, mixtures of hexane and ethyl acetate).

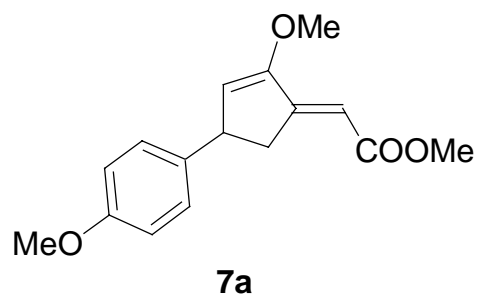

Compound 7a. The general procedure was followed using complex 1a (184 mg, 0.5 $\mathrm{mmol})$, allene 3a (147 mg, $1.5 \mathrm{mmol})$ and $[\mathrm{Rh}($ naphthalene $)(\mathrm{cod})]\left[\mathrm{SbF}_{6}\right](29 \mathrm{mg}, 0.05$ mmol) in $\mathrm{CH}_{2} \mathrm{Cl}_{2}(10 \mathrm{~mL})$. Final chromatographic purification using a 5:1 mixture of hexane:ethyl acetate as eluent afforded 7a $(99 \mathrm{mg}, 72 \%) .{ }^{1} \mathrm{H}-\mathrm{NMR}\left(\mathrm{CDCl}_{3}\right): 2.89$ (dt, $J$ $=19.7$ and $2.4 \mathrm{~Hz}, 1 \mathrm{H}), 3.58(\mathrm{ddd}, J=19.7,7.0$ and $2.4 \mathrm{~Hz}, 1 \mathrm{H}), 3.72(\mathrm{~s}, 3 \mathrm{H}), 3.78(\mathrm{~s}$, 3H), $3.81(\mathrm{~s}, 3 \mathrm{H}), 3.97(\mathrm{~m}, 1 \mathrm{H}), 5.49(\mathrm{~d}, J=2.8 \mathrm{~Hz}, 1 \mathrm{H}), 5.96(\mathrm{t}, J=2.4 \mathrm{~Hz}, 1 \mathrm{H}), 6.85$ $(\mathrm{d}, J=8.7 \mathrm{~Hz}, 2 \mathrm{H}), 7.12(\mathrm{~d}, J=8.7 \mathrm{~Hz}, 2 \mathrm{H}) ;{ }^{13} \mathrm{C}-\mathrm{NMR}\left(\mathrm{CDCl}_{3}\right): 39.3\left(\mathrm{CH}_{2}\right), 44.8(\mathrm{CH})$, $50.9\left(\mathrm{CH}_{3}\right), 55.2\left(\mathrm{CH}_{3}\right), 56.9\left(\mathrm{CH}_{3}\right), 106.6(\mathrm{CH}), 113.9(\mathrm{CH}), 116.1(\mathrm{CH}), 127.8(\mathrm{CH})$, 137.1 (C), 158.2 (C), 158.3 (C), 158.7 (C), 167.9 (C). IR $\left(\mathrm{CH}_{2} \mathrm{Cl}_{2}\right)$ : v 1703, 1634, 1606, 1511, $1100 \mathrm{~cm}^{-1}$. Anal. Calcd for $\mathrm{C}_{16} \mathrm{H}_{18} \mathrm{O}_{4}: \mathrm{C}, 70.06$; H, 6.61; Found: C, 70.14; H, 6.59 . 


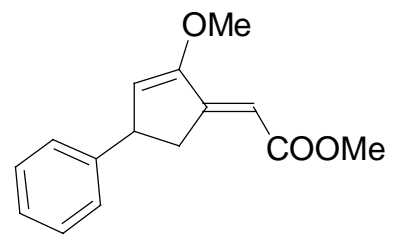

$7 b$

Compound 7b. The general procedure was followed using complex $\mathbf{1 b}$ (169 $\mathrm{mg}, 0.5$ $\mathrm{mmol})$, allene $3 \mathbf{a}(147 \mathrm{mg}, 1.5 \mathrm{mmol})$ and $[\mathrm{Rh}($ naphthalene)(cod)][SbF 6$](29 \mathrm{mg}, 0.05$ mmol) in $\mathrm{CH}_{2} \mathrm{Cl}_{2}(10 \mathrm{~mL})$. Final chromatographic purification using a 5:1 mixture of hexane:ethyl acetate as eluent afforded $7 \mathbf{b}(80 \mathrm{mg}, 66 \%) .{ }^{1} \mathrm{H}-\mathrm{NMR}\left(\mathrm{CDCl}_{3}\right): 2.93(\mathrm{dt}, J$ $=19.7$ and $2.2 \mathrm{~Hz}, 1 \mathrm{H}), 3.58-3.72(\mathrm{~m}, 1 \mathrm{H}), 3.72(\mathrm{~s}, 3 \mathrm{H}), 3.79(\mathrm{~s}, 3 \mathrm{H}), 4.01-4.03(\mathrm{~m}$, $1 \mathrm{H}), 5.51(\mathrm{~d}, J=2.8 \mathrm{~Hz}, 1 \mathrm{H}), 5.97(\mathrm{t}, J=2.4 \mathrm{~Hz}, 1 \mathrm{H}), 7.16-7.22(\mathrm{~m}, 5 \mathrm{H}) ;{ }^{13} \mathrm{C}-\mathrm{NMR}$ $\left(\mathrm{CDCl}_{3}\right): 39.1\left(\mathrm{CH}_{2}\right), 45.6(\mathrm{CH}), 51.0\left(\mathrm{CH}_{3}\right), 57.0\left(\mathrm{CH}_{3}\right), 106.7(\mathrm{CH}), 115.8(\mathrm{CH})$, $126.5(\mathrm{CH}), 126.9(\mathrm{CH}), 128.5(\mathrm{CH}), 145.0(\mathrm{C}), 158.6(\mathrm{C}), 167.9(\mathrm{C})$. Anal. Calcd for $\mathrm{C}_{15} \mathrm{H}_{16} \mathrm{O}_{3}:$ C, 73.75; H, 6.60; Found: C, 73.66; H, 6.63.

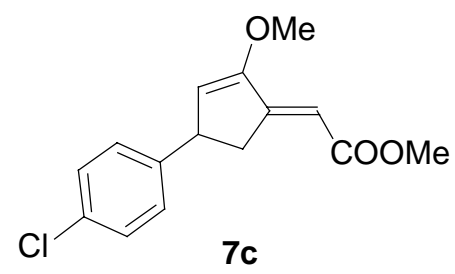

Compound 7c. The general procedure was followed using complex 1c (186 mg, 0.5 $\mathrm{mmol})$, allene $3 \mathbf{a}(147 \mathrm{mg}, 1.5 \mathrm{mmol})$ and $[\mathrm{Rh}$ (naphthalene)(cod)][SbF 6$](29 \mathrm{mg}, 0.05$ mmol) in $\mathrm{CH}_{2} \mathrm{Cl}_{2}(10 \mathrm{~mL})$. Final chromatographic purification using a 5:1 mixture of hexane:ethyl acetate as eluent afforded 7c $(86 \mathrm{mg}, 62 \%) .{ }^{1} \mathrm{H}-\mathrm{NMR}\left(\mathrm{CDCl}_{3}\right): 2.86(\mathrm{dt}, J$ $=19.5$ and $2.4 \mathrm{~Hz}, 1 \mathrm{H}), 3.58(\mathrm{ddd}, J=19.5,7.0$ and $2.4 \mathrm{~Hz}, 1 \mathrm{H}), 3.72(\mathrm{~s}, 3 \mathrm{H}), 3.79$ (s, 3H), 3.96-4.00 (m, 1H), 5.45 (d, $J=2.6 \mathrm{~Hz}, 1 \mathrm{H}), 5.96$ (t, $J=2.4 \mathrm{~Hz}, 1 \mathrm{H}), 7.12(\mathrm{~d}, J=$ $8.8 \mathrm{~Hz}, 2 \mathrm{H}), 7.26(\mathrm{~d}, J=8.8 \mathrm{~Hz}, 2 \mathrm{H}) ;{ }^{13} \mathrm{C}-\mathrm{NMR}\left(\mathrm{CDCl}_{3}\right): 39.0\left(\mathrm{CH}_{2}\right), 45.0(\mathrm{CH}), 51.0$ $\left(\mathrm{CH}_{3}\right), 57.0\left(\mathrm{CH}_{3}\right), 107.1(\mathrm{CH}), 115.1(\mathrm{CH}), 128.3(\mathrm{CH}), 128.6(\mathrm{CH}), 132.2(\mathrm{C}), 143.6$ 
(C), 158.1 (C), 158.9 (C), 167.8 (C). Anal. Calcd for $\mathrm{C}_{15} \mathrm{H}_{15} \mathrm{ClO}_{3}$ : C, 64.64; $\mathrm{H}, 5.42$;

Found: C, 64.42; H, 5.37.
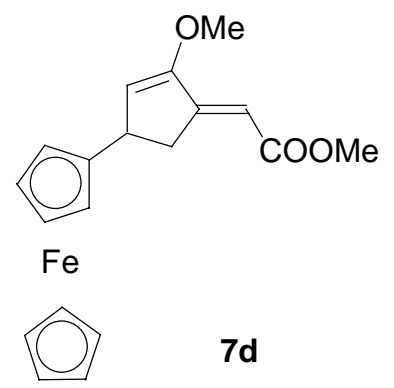

Compound 7d. The general procedure was followed using complex 1e ( $223 \mathrm{mg}, 0.5$ $\mathrm{mmol})$, allene 3a (147 mg, $1.5 \mathrm{mmol})$ and $[\mathrm{Rh}($ naphthalene $)(\mathrm{cod})]\left[\mathrm{SbF}_{6}\right](29 \mathrm{mg}, 0.05$ mmol) in $\mathrm{CH}_{2} \mathrm{Cl}_{2}(10 \mathrm{~mL})$. Final chromatographic purification using a 5:1 mixture of hexane:ethyl acetate as eluent afforded 7d $(84 \mathrm{mg}, 48 \%) .{ }^{1} \mathrm{H}-\mathrm{NMR}\left(\mathrm{CDCl}_{3}\right): 3.02(\mathrm{dt}, J$ $=19.4$ and $2.2 \mathrm{~Hz}, 1 \mathrm{H}), 3.46(\mathrm{ddd}, J=19.4,6.6$ and $2.2 \mathrm{~Hz}, 1 \mathrm{H}), 3.73(\mathrm{~s}, 3 \mathrm{H}), 3.76$ (s, $3 \mathrm{H}), 4.02-4.14(\mathrm{~m}, 10 \mathrm{H}), 5.51(\mathrm{~d}, J=2.6 \mathrm{~Hz}, 1 \mathrm{H}), 5.90(\mathrm{br} \mathrm{s}, 1 \mathrm{H}) ;{ }^{13} \mathrm{C}-\mathrm{NMR}\left(\mathrm{CDCl}_{3}\right)$ : $37.8\left(\mathrm{CH}_{2}\right), 39.4(\mathrm{CH}), 51.0\left(\mathrm{CH}_{3}\right), 56.8\left(\mathrm{CH}_{3}\right), 66.3(\mathrm{CH}), 66.8(\mathrm{CH}), 67.3(\mathrm{CH}), 67.6$ $(\mathrm{CH}), 68.3(\mathrm{CH}), 92.9(\mathrm{C}), 106.3(\mathrm{CH}), 116.1(\mathrm{CH}), 157.6(\mathrm{C}), 158.9(\mathrm{C}), 168.0(\mathrm{C})$. Anal. Calcd for $\mathrm{C}_{19} \mathrm{H}_{20} \mathrm{FeO}_{3}$ : C, 64.79; H, 5.72; Found: C, 64.91; H, 5.71.

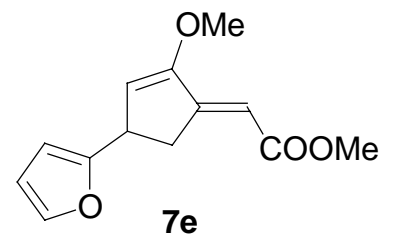

Compound 7e. The general procedure was followed using complex 1d (164 mg, 0.5 $\mathrm{mmol})$, allene 3a (147 mg, $1.5 \mathrm{mmol})$ and $[\mathrm{Rh}($ naphthalene $)(\mathrm{cod})]\left[\mathrm{SbF}_{6}\right](29 \mathrm{mg}, 0.05$ mmol) in $\mathrm{CH}_{2} \mathrm{Cl}_{2}(10 \mathrm{~mL})$. Final chromatographic purification using a 5:1 mixture of hexane:ethyl acetate as eluent afforded 7e $(69 \mathrm{mg}, 59 \%) .{ }^{1} \mathrm{H}-\mathrm{NMR}\left(\mathrm{CDCl}_{3}\right): 3.06(\mathrm{dt}, \mathrm{J}$ $=19.6$ and $2.4 \mathrm{~Hz}, 1 \mathrm{H}), 3.47(\mathrm{ddd}, \mathrm{J}=19.6,7.2$ and $2.4 \mathrm{~Hz}, 1 \mathrm{H}), 3.71(\mathrm{~s}, 3 \mathrm{H}), 3.75$ (s, 3H), 4.04-4.09 (m, 1H), $5.46(\mathrm{~d}, \mathrm{~J}=2,8 \mathrm{~Hz}, 1 \mathrm{H}), 5.93(\mathrm{t}, \mathrm{J}=2.4 \mathrm{~Hz}, 1 \mathrm{H}), 6.01(\mathrm{~d}, \mathrm{~J}=$ $3.1 \mathrm{~Hz}, 1 \mathrm{H}), 6.27(\mathrm{dd}, \mathrm{J}=3.1$ and $1.9 \mathrm{~Hz}, 1 \mathrm{H}), 7.31(\mathrm{dd}, \mathrm{J}=1.9$ and $0.9 \mathrm{~Hz}, 1 \mathrm{H}) ;{ }^{13} \mathrm{C}-$ 
NMR $\left(\mathrm{CDCl}_{3}\right): 35.4\left(\mathrm{CH}_{2}\right), 38.9(\mathrm{CH}), 51.0\left(\mathrm{CH}_{3}\right), 56.9\left(\mathrm{CH}_{3}\right), 104.2(\mathrm{CH}), 107.1$ (CH), $110.1(\mathrm{CH}), 112.3(\mathrm{CH}), 141.4(\mathrm{CH}), 157.2(\mathrm{C}), 157.5(\mathrm{C}), 158.7(\mathrm{C}), 167.8(\mathrm{C})$. Anal. Calcd for $\mathrm{C}_{13} \mathrm{H}_{14} \mathrm{O}_{4}$ : C, 66.66; H, 6.02; Found: C, 66.49; H, 6.00.

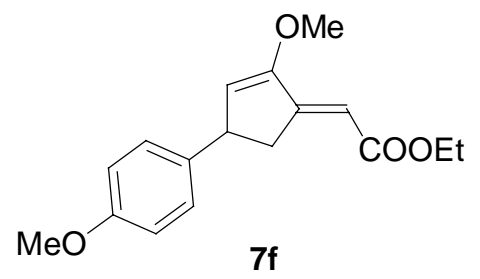

Compound 7f. The general procedure was followed using complex 1a (184 mg, 0.5 mmol), allene $3 \mathbf{b}(168 \mathrm{mg}, 1.5 \mathrm{mmol})$ and $[\mathrm{Rh}($ naphthalene $)(\mathrm{cod})]\left[\mathrm{SbF}_{6}\right](29 \mathrm{mg}, 0.05$ mmol) in $\mathrm{CH}_{2} \mathrm{Cl}_{2}(10 \mathrm{~mL})$. Final chromatographic purification using a 5:1 mixture of hexane:ethyl acetate as eluent afforded $7 \mathbf{f}(75 \mathrm{mg}, 52 \%) .{ }^{1} \mathrm{H}-\mathrm{NMR}\left(\mathrm{CDCl}_{3}\right): 1.28(\mathrm{t}, \mathrm{J}=$ $7.0 \mathrm{~Hz}, 3 \mathrm{H}), 2.86(\mathrm{dt}, J=19.6$ and $2.4 \mathrm{~Hz}, 1 \mathrm{H}), 3.56(\mathrm{ddd}, J=19.6,7.0$ and $2.4 \mathrm{~Hz}$, 1H), $3.78(\mathrm{~s}, 3 \mathrm{H}), 3.81(\mathrm{~s}, 3 \mathrm{H}), 3.94-3.98(\mathrm{~m}, 1 \mathrm{H}), 4.17(\mathrm{q}, J=7.0 \mathrm{~Hz}, 2 \mathrm{H}), 5.46(\mathrm{~d}, J=$ $2.9 \mathrm{~Hz}, 1 \mathrm{H}), 5.94(\mathrm{t}, J=2.4 \mathrm{~Hz}, 1 \mathrm{H}), 6.85(\mathrm{~d}, J=8.7 \mathrm{~Hz}, 2 \mathrm{H}), 7.11(\mathrm{~d}, J=8.7 \mathrm{~Hz}, 2 \mathrm{H})$; ${ }^{13} \mathrm{C}-\mathrm{NMR}\left(\mathrm{CDCl}_{3}\right): 14.4\left(\mathrm{CH}_{3}\right), 39.2\left(\mathrm{CH}_{2}\right), 44.8(\mathrm{CH}), 55.3\left(\mathrm{CH}_{3}\right), 56.9\left(\mathrm{CH}_{3}\right), 59.6$ $\left(\mathrm{CH}_{2}\right), 107.1(\mathrm{CH}), 113.9(\mathrm{CH}), 115.9(\mathrm{CH}), 127.9(\mathrm{CH}), 137.2(\mathrm{C}), 158.2(\mathrm{C}), 158.39$ (C), $158.41(\mathrm{C}), 167.5(\mathrm{C})$. IR $\left(\mathrm{CH}_{2} \mathrm{Cl}_{2}\right): v 1696,1634,1606,1421 \mathrm{~cm}^{-1}$. Anal. Calcd for $\mathrm{C}_{17} \mathrm{H}_{20} \mathrm{O}_{4}$ : C, 70.81; H, 6.99; Found: C, 70.96; H, 6.83.

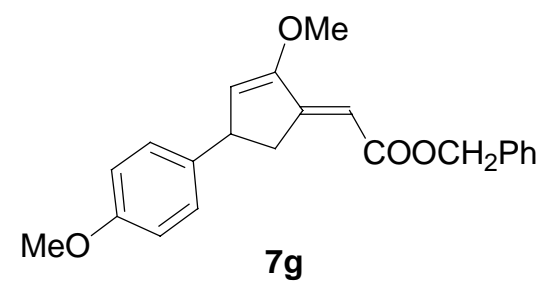

Compound 7g. The general procedure was followed using complex 1a (184 mg, 0.5 $\mathrm{mmol})$, allene 3c (174 mg, 1mmol) and $[\mathrm{Rh}($ naphthalene $)(\mathrm{cod})]\left[\mathrm{SbF}_{6}\right](29 \mathrm{mg}, 0.05$ mmol) in $\mathrm{CH}_{2} \mathrm{Cl}_{2}(10 \mathrm{~mL})$. Final chromatographic purification using a 5:1 mixture of hexane:ethyl acetate as eluent afforded $7 \mathrm{~g}(98 \mathrm{mg}, 56 \%) .{ }^{1} \mathrm{H}-\mathrm{NMR}\left(\mathrm{CDCl}_{3}\right): 2.90(\mathrm{dt}, \mathrm{J}$ 
$=19.8$ and $2.1 \mathrm{~Hz}, 1 \mathrm{H}), 3.58(\mathrm{ddd}, \mathrm{J}=19.8,6.9$ and $2.1 \mathrm{~Hz}, 1 \mathrm{H}), 3.76(\mathrm{~s}, 3 \mathrm{H}), 3.79(\mathrm{~s}$, 3H), 3.95-3.97 (m, 1H), 5.16 (s, 2H), 5.47 (d, J = 2.7 Hz, 1H), 6.01 (br s, 1H), 6.83 (d, J $=8.8 \mathrm{~Hz}, 2 \mathrm{H}), 7.11(\mathrm{~d}, \mathrm{~J}=8.8 \mathrm{~Hz}, 2 \mathrm{H}), 7.32-7.37(\mathrm{~m}, 5 \mathrm{H}) ;{ }^{13} \mathrm{C}-\mathrm{NMR}\left(\mathrm{CDCl}_{3}\right): 39.3$ $\left(\mathrm{CH}_{2}\right), 44.8(\mathrm{CH}), 55.2\left(\mathrm{CH}_{3}\right), 56.9\left(\mathrm{CH}_{3}\right), 65.5\left(\mathrm{CH}_{2}\right), 106.7(\mathrm{CH}), 113.9(\mathrm{CH}), 116.3$ $(\mathrm{CH}), 127.9(\mathrm{CH}), 128.0(\mathrm{CH}), 128.4(\mathrm{CH}), 136.5(\mathrm{C}), 137.0(\mathrm{C}), 158.2(\mathrm{C}), 158.3(\mathrm{C})$, 159.2 (C), 167.2 (C). Anal. Calcd for $\mathrm{C}_{22} \mathrm{H}_{22} \mathrm{O}_{4}: \mathrm{C}, 75.41 ; \mathrm{H}, 6.33$; Found: $\mathrm{C}, 75.53 ; \mathrm{H}$, 6.41.

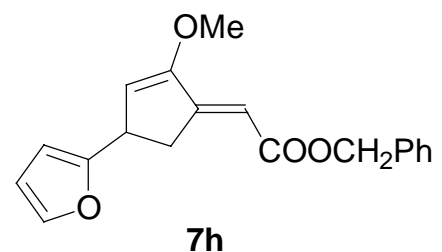

Compound 7h. The general procedure was followed using complex 1d (164 mg, 0.5 $\mathrm{mmol})$, allene 3c $(174 \mathrm{mg}, 1 \mathrm{mmol})$ and $[\mathrm{Rh}($ naphthalene $)(\mathrm{cod})]\left[\mathrm{SbF}_{6}\right](29 \mathrm{mg}, 0.05$ mmol) in $\mathrm{CH}_{2} \mathrm{Cl}_{2}(10 \mathrm{~mL})$. Final chromatographic purification using a 5:1 mixture of hexane:ethyl acetate as eluent afforded $7 \mathbf{h}(84 \mathrm{mg}, 54 \%) .{ }^{1} \mathrm{H}-\mathrm{NMR}\left(\mathrm{CDCl}_{3}\right): 3.11$ (dt, J $=19.5$ and $2.4 \mathrm{~Hz}, 1 \mathrm{H}), 3.51(\mathrm{ddd}, \mathrm{J}=19.5,7.0$, and $2.4 \mathrm{~Hz}, 1 \mathrm{H}), 3.77(\mathrm{~s}, 3 \mathrm{H}), 4.08-$ $4.10(\mathrm{~m}, 1 \mathrm{H}), 5.19(\mathrm{~s}, 2 \mathrm{H}), 5.50(\mathrm{~d}, \mathrm{~J}=2.8 \mathrm{~Hz}, 1 \mathrm{H}), 6.01-6.04(\mathrm{~m}, 2 \mathrm{H}), 6.29-6.31(\mathrm{~m}$, 1H), 7.28-7.39 (m, 6H); ${ }^{13} \mathrm{C}-\mathrm{NMR}\left(\mathrm{CDCl}_{3}\right): 35.4\left(\mathrm{CH}_{2}\right), 38.9(\mathrm{CH}), 56.9\left(\mathrm{CH}_{3}\right), 65.5$ $\left(\mathrm{CH}_{2}\right), 104.2(\mathrm{CH}), 107.1(\mathrm{CH}), 110.1(\mathrm{CH}), 112.4(\mathrm{CH}), 127.9(\mathrm{CH}), 128.1(\mathrm{CH})$, $128.4(\mathrm{CH}), 136.4(\mathrm{C}), 141.3(\mathrm{CH}), 157.1(\mathrm{C}), 157.9$ (C), $158.6(\mathrm{C}), 167.1(\mathrm{C})$. Anal. Calcd for $\mathrm{C}_{19} \mathrm{H}_{18} \mathrm{O}_{4}$ : C, 73.53; H, 5.85; Found: C, 73.58; H, 5.77.

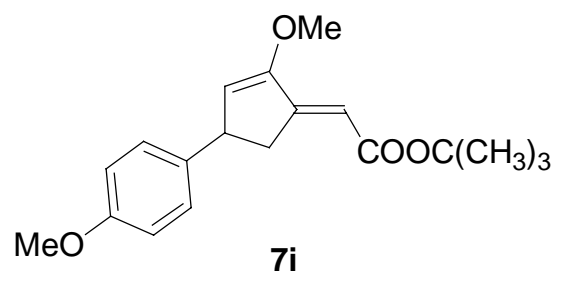

Compound 7i. The general procedure was followed using complex 1a (184 mg, 0.5 $\mathrm{mmol})$, allene $3 \mathbf{d}(210 \mathrm{mg}, 1.5 \mathrm{mmol})$ and $[\mathrm{Rh}($ naphthalene)(cod)][SbF 6$](29 \mathrm{mg}, 0.05$ 
mmol) in $\mathrm{CH}_{2} \mathrm{Cl}_{2}(10 \mathrm{~mL})$. Final chromatographic purification using a 5:1 mixture of hexane:ethyl acetate as eluent afforded $7 \mathbf{i}(81 \mathrm{mg}, 51 \%) .{ }^{1} \mathrm{H}-\mathrm{NMR}\left(\mathrm{CDCl}_{3}\right): 1.46(\mathrm{~s}$, 9H), $2.81(\mathrm{dt}, \mathrm{J}=19.6$ and $2.3 \mathrm{~Hz}, 1 \mathrm{H}), 3.53(\mathrm{ddd}, \mathrm{J}=19.7,7.1$, and $2.5 \mathrm{~Hz}, 1 \mathrm{H}), 3.74$ (s, 3H), $3.78(\mathrm{~s}, 3 \mathrm{H}), 3.90-3.93(\mathrm{~m}, 1 \mathrm{H}), 5.40(\mathrm{~d}, \mathrm{~J}=2.8 \mathrm{~Hz}, 1 \mathrm{H}), 5.84(\mathrm{~d}, \mathrm{~J}=2.4 \mathrm{~Hz}$, 1H), $6.82(\mathrm{~d}, \mathrm{~J}=8.4 \mathrm{~Hz}, 2 \mathrm{H}), 7.09(\mathrm{~d}, \mathrm{~J}=8.4 \mathrm{~Hz}, 2 \mathrm{H}) ;{ }^{13} \mathrm{C}-\mathrm{NMR}\left(\mathrm{CDCl}_{3}\right): 28.4\left(\mathrm{CH}_{3}\right)$, $39.1\left(\mathrm{CH}_{2}\right), 44.8\left(\mathrm{CH}_{2}\right), 55.3\left(\mathrm{CH}_{3}\right), 56.9\left(\mathrm{CH}_{3}\right), 79.6(\mathrm{C}), 109.1(\mathrm{CH}), 113.9(\mathrm{CH})$, $115.2(\mathrm{CH}), 127.9(\mathrm{CH}), 137.4(\mathrm{C}), 157.1(\mathrm{C}), 158.2(\mathrm{C}), 158.6(\mathrm{C}), 167.2(\mathrm{C})$. Anal. Calcd for $\mathrm{C}_{19} \mathrm{H}_{24} \mathrm{O}_{4}$ : C, 72.13; H, 7.65; Found: C, 71.98; H, 7.73.

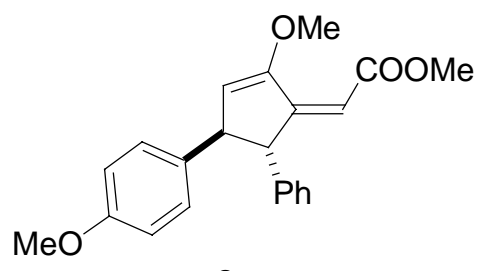

$8 \mathrm{a}$

Compound 8a. The general procedure was followed using complex 1a (184 mg, 0.5 $\mathrm{mmol})$, allene $3 \mathbf{e}(174 \mathrm{mg}, 1 \mathrm{mmol})$ and $[\mathrm{Rh}($ naphthalene $)(\mathrm{cod})]\left[\mathrm{SbF}_{6}\right](29 \mathrm{mg}, 0.05$ mmol) in $\mathrm{CH}_{2} \mathrm{Cl}_{2}(10 \mathrm{~mL})$. Final chromatographic purification using a 5:1 mixture of hexane:ethyl acetate as eluent afforded 8a (122 mg, $70 \%) .{ }^{1} \mathrm{H}-\mathrm{NMR}\left(\mathrm{CDCl}_{3}\right): 3.69$ (dd, $\mathrm{J}=3.2$ and $2.7 \mathrm{~Hz}, 1 \mathrm{H}), 3.72(\mathrm{~s}, 3 \mathrm{H}), 3.79(\mathrm{~s}, 3 \mathrm{H}), 3.82(\mathrm{~s}, 3 \mathrm{H}), 3.89(\mathrm{t}, \mathrm{J}=3.2 \mathrm{~Hz}, 1 \mathrm{H})$, 5.32 (br s, 1H), 5.39-5.40 (m, 1H), $6.83(\mathrm{~d}, \mathrm{~J}=8.5 \mathrm{~Hz}, 2 \mathrm{H}), 7.03(\mathrm{~d}, \mathrm{~J}=8.5 \mathrm{~Hz}, 2 \mathrm{H})$, 7.12-7.15 (m, 2H), 7.25-7.33 (m, 3H); ${ }^{13} \mathrm{C}-\mathrm{NMR}\left(\mathrm{CDCl}_{3}\right): 51.4\left(\mathrm{CH}_{3}\right), 54.3(\mathrm{CH}), 55.2$ $\left(\mathrm{CH}_{3}\right), 57.2\left(\mathrm{CH}_{3}\right), 59.4(\mathrm{CH}), 111.4(\mathrm{CH}), 112.8(\mathrm{CH}), 113.8(\mathrm{CH}), 126.8(\mathrm{CH}), 128.0$ (CH), $128.4(\mathrm{CH}), 128.6(\mathrm{CH}), 136.0(\mathrm{C}), 142.1(\mathrm{C}), 152.9(\mathrm{C}), 157.7(\mathrm{C}), 158.4(\mathrm{C})$, 167.4 (C). IR $\left(\mathrm{CH}_{2} \mathrm{Cl}_{2}\right): v 1721,1610,1470,1384 \mathrm{~cm}^{-1}$. Anal. Calcd for $\mathrm{C}_{22} \mathrm{H}_{22} \mathrm{O}_{4}: \mathrm{C}$, 75.41; H, 6.33; Found: C, 75.61; H, 6.17. 


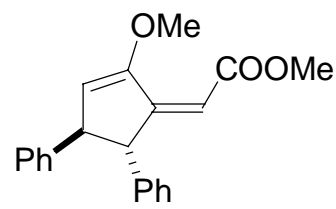

$8 b$

Compound 8b. The general procedure was followed using complex $\mathbf{1 b}$ (169 $\mathrm{mg}, 0.5$ $\mathrm{mmol})$, allene $3 \mathbf{e}(174 \mathrm{mg}, 1 \mathrm{mmol})$ and $[\mathrm{Rh}($ naphthalene $)(\mathrm{cod})]\left[\mathrm{SbF}_{6}\right](29 \mathrm{mg}, 0.05$ mmol) in $\mathrm{CH}_{2} \mathrm{Cl}_{2}(10 \mathrm{~mL})$. Final chromatographic purification using a 5:1 mixture of hexane:ethyl acetate as eluent afforded $8 \mathbf{b}(106 \mathrm{mg}, 66 \%) .{ }^{1} \mathrm{H}-\mathrm{NMR}\left(\mathrm{CDCl}_{3}\right)$ : 3.69$3.71(\mathrm{~s}+\mathrm{m}, 4 \mathrm{H}), 3.83(\mathrm{~s}, 3 \mathrm{H}), 3.92(\mathrm{t}, \mathrm{J}=2.9 \mathrm{~Hz}, 1 \mathrm{H}), 5.30($ br s, 1H), 5.41 (br s, 1H), 7.09-7.26 (m, 10H); ${ }^{13} \mathrm{C}-\mathrm{NMR}\left(\mathrm{CDCl}_{3}\right): 51.4\left(\mathrm{CH}_{3}\right), 55.0(\mathrm{CH}), 57.2\left(\mathrm{CH}_{3}\right), 59.1(\mathrm{CH})$, $111.5(\mathrm{CH}), 112.4(\mathrm{CH}), 126.7(\mathrm{CH}), 126.9(\mathrm{CH}), 127.0(\mathrm{CH}), 128.4(\mathrm{CH}), 128.5(\mathrm{CH})$, $128.6(\mathrm{CH}), 142.1(\mathrm{C}), 143.9(\mathrm{C}), 152.8(\mathrm{C}), 157.9(\mathrm{C}), 167.3(\mathrm{C}) . \mathrm{IR}\left(\mathrm{CH}_{2} \mathrm{Cl}_{2}\right):$ v 1732, $1614 \mathrm{~cm}^{-1}$.Anal. Calcd for $\mathrm{C}_{21} \mathrm{H}_{20} \mathrm{O}_{3}$ : C, 78.73; H, 6.29; Found: C, 78.75; H, 6.34.

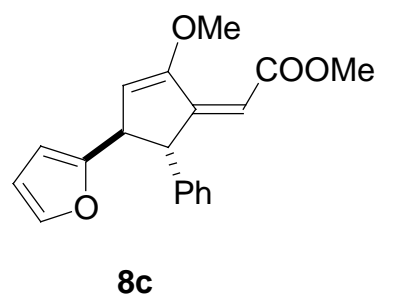

Compound 8c. The general procedure was followed using complex 1d (164 mg, 0.5 $\mathrm{mmol})$, allene $3 \mathbf{e}(174 \mathrm{mg}, 1 \mathrm{mmol})$ and $[\mathrm{Rh}($ naphthalene $)(\mathrm{cod})]\left[\mathrm{SbF}_{6}\right](29 \mathrm{mg}, 0.05$ mmol) in $\mathrm{CH}_{2} \mathrm{Cl}_{2}(10 \mathrm{~mL})$. Final chromatographic purification using a 5:1 mixture of hexane:ethyl acetate as eluent afforded 8c $(101 \mathrm{mg}, 65 \%) .{ }^{1} \mathrm{H}-\mathrm{NMR}\left(\mathrm{CDCl}_{3}\right): 3.71$ (s, 3H), 3.80 (s, 3H), 3.98-4.02 (m, 2H), 5.35 (br s, 1H), 5.38 (d, J = 1.6 Hz, 1H), 6.03 (d, J $=3.2 \mathrm{~Hz}, 1 \mathrm{H}), 6.29(\mathrm{t}, \mathrm{J}=2.4 \mathrm{~Hz}, 1 \mathrm{H}), 7.20-7.35(\mathrm{~m}, 6 \mathrm{H}) ;{ }^{13} \mathrm{C}-\mathrm{NMR}\left(\mathrm{CDCl}_{3}\right): 48.3$ $(\mathrm{CH}), 51.4\left(\mathrm{CH}_{3}\right), 55.0(\mathrm{CH}), 57.2\left(\mathrm{CH}_{3}\right), 105.0(\mathrm{CH}), 109.3(\mathrm{CH}), 110.1(\mathrm{CH}), 112.0$ $(\mathrm{CH}), 127.0(\mathrm{CH}), 128.3(\mathrm{CH}), 128.7(\mathrm{CH}), 141.6(\mathrm{CH}), 142.1(\mathrm{C}), 151.7(\mathrm{C}), 156.3$ 
(C), 157.9 (C), 167.3 (C). IR $\left(\mathrm{CH}_{2} \mathrm{Cl}_{2}\right): v$ 1727, 1614, $1454 \mathrm{~cm}^{-1}$. Anal. Calcd for $\mathrm{C}_{19} \mathrm{H}_{18} \mathrm{O}_{4}$ : C, 73.53; H, 5.85; Found: C, 73.62; H, 5.92.

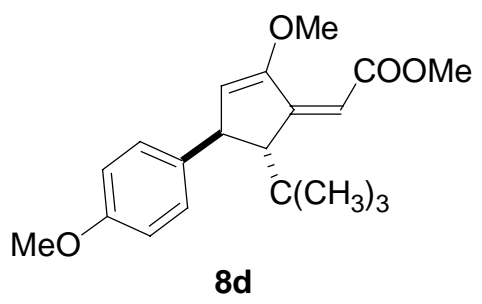

Compounds 8d. The general procedure was followed using complex 1a (184 mg, 0.5 mmol), allene 3f (154 mg, 1mmol) and [Rh(naphthalene)(cod)][SbF 6 (29 mg, 0.05 mmol) in $\mathrm{CH}_{2} \mathrm{Cl}_{2}(10 \mathrm{~mL})$. Final chromatographic purification using a 5:1 mixture of hexane:ethyl acetate as eluent afforded 8d (109 mg, $66 \%) .{ }^{1} \mathrm{H}-\mathrm{NMR}\left(\mathrm{CDCl}_{3}\right): 0.98$ (s, 9H), 2.32 (br s, 1H), $3.658 \mathrm{~d}, \mathrm{~J}=3.1 \mathrm{~Hz}, 1 \mathrm{H}), 3.70(\mathrm{~s}, 3 \mathrm{H}), 3.76(\mathrm{~s}, 3 \mathrm{H}), 3.79(\mathrm{~s}, 3 \mathrm{H})$, $5.25(\mathrm{~d}, \mathrm{~J}=2.0 \mathrm{~Hz}, 1 \mathrm{H}), 5.66(\mathrm{br} \mathrm{s}, 1 \mathrm{H}), 6.82(\mathrm{~d}, \mathrm{~J}=8.7 \mathrm{~Hz}, 2 \mathrm{H}), 7.12(\mathrm{~d}, \mathrm{~J}=8.7 \mathrm{~Hz}$, $2 \mathrm{H}) ;{ }^{13} \mathrm{C}-\mathrm{NMR}\left(\mathrm{CDCl}_{3}\right): 27.3\left(\mathrm{CH}_{3}\right), 34.6(\mathrm{C}), 47.0(\mathrm{CH}), 51.4\left(\mathrm{CH}_{3}\right), 55.2\left(\mathrm{CH}_{3}\right), 57.1$ $\left(\mathrm{CH}_{3}\right), 62.3(\mathrm{CH}), 112.9(\mathrm{CH}), 113.3(\mathrm{CH}), 113.9(\mathrm{CH}), 128.0(\mathrm{C}), 137.5(\mathrm{C}), 149.7(\mathrm{C})$, 158.09 (C), $158.13(\mathrm{C}), 167.4(\mathrm{C}) . \mathrm{IR}\left(\mathrm{CH}_{2} \mathrm{Cl}_{2}\right): v 1722,1615,1510,1175 \mathrm{~cm}^{-1}$. Anal. Calcd for $\mathrm{C}_{20} \mathrm{H}_{26} \mathrm{O}_{4}$ : C, 72.70; H, 7.93; Found: C, 72.73; H, 7.93.

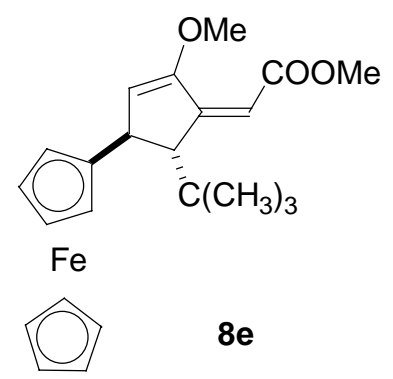

Compound 8e. The general procedure was followed using complex 1e (223 mg, 0.5 $\mathrm{mmol})$, allene $3 f(154 \mathrm{mg}, 1 \mathrm{mmol})$ and $[\mathrm{Rh}($ naphthalene $)(\mathrm{cod})]\left[\mathrm{SbF}_{6}\right](29 \mathrm{mg}, 0.05$ mmol) in $\mathrm{CH}_{2} \mathrm{Cl}_{2}(10 \mathrm{~mL})$. Final chromatographic purification using a 5:1 mixture of hexane:ethyl acetate as eluent afforded $8 \mathbf{e}(126 \mathrm{mg}, 62 \%) .{ }^{1} \mathrm{H}-\mathrm{NMR}\left(\mathrm{CDCl}_{3}\right): 0.94$ (s, 9H), 2.16 (br s, 1H), 3.38 (d, J = 3.5 Hz, 1H), $3.71(\mathrm{~s}, 3 \mathrm{H}), 3.76(\mathrm{~s}, 3 \mathrm{H}), 3.94-3.95$ (m, 
$1 \mathrm{H}), 4.01-4.10(\mathrm{~m}, 3 \mathrm{H}), 4.14(\mathrm{~s}, 5 \mathrm{H}), 5.39-5.41(\mathrm{~m}, 1 \mathrm{H}), 5.56(\mathrm{br} \mathrm{s}, 1 \mathrm{H}) ;{ }^{13} \mathrm{C}-\mathrm{NMR}$ $\left(\mathrm{CDCl}_{3}\right): 27.3\left(\mathrm{CH}_{3}\right), 34.2(\mathrm{C}), 41.5(\mathrm{CH}), 51.3\left(\mathrm{CH}_{3}\right), 57.1\left(\mathrm{CH}_{3}\right), 62.2(\mathrm{CH}), 66.2$ $(\mathrm{CH}), 67.0(\mathrm{CH}), 67.2(\mathrm{CH}), 67.4(\mathrm{CH}), 68.3(\mathrm{CH}), 93.9(\mathrm{C}), 111.5(\mathrm{CH}), 113.4(\mathrm{CH})$, 149.6 (C), 158.1 (C), 167.3 (C). Anal. Calcd for $\mathrm{C}_{23} \mathrm{H}_{28} \mathrm{FeO}_{3}$ : C, 67.66; H, 6.91; Found: C, 67.85; H, 6.78.

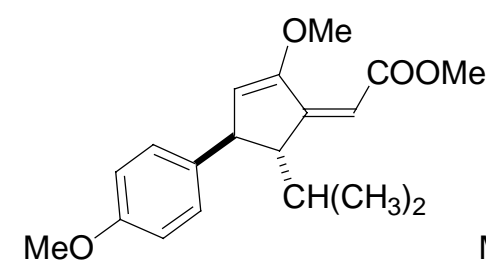

$8 f$

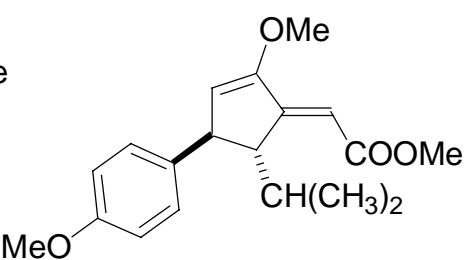

9f

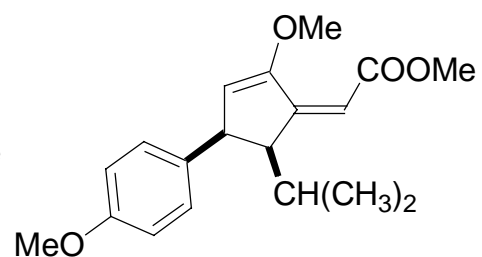

$10 f$

Compounds 8f, 9f and 10f. The general procedure was followed using complex 1a (184 mg, $0.5 \mathrm{mmol})$, allene $3 \mathrm{~g}(210 \mathrm{mg}, 1.5 \mathrm{mmol})$ and $[\mathrm{Rh}($ naphthalene $)(\mathrm{cod})]\left[\mathrm{SbF}_{6}\right]$ (29 mg, $0.05 \mathrm{mmol})$ in $\mathrm{CH}_{2} \mathrm{Cl}_{2}(10 \mathrm{~mL})$. Final chromatographic purification using a 5:1 mixture of hexane:ethyl acetate as eluent afforded $\mathbf{8 f}(107 \mathrm{mg}, 68 \%)$ and a 1:1 mixture of 9f and 10f (16 mg, 10\%). Compound 8f. ${ }^{1} \mathrm{H}-\mathrm{NMR}\left(\mathrm{CDCl}_{3}\right): 0.88(\mathrm{~d}, \mathrm{~J}=6.8 \mathrm{~Hz}, 3 \mathrm{H})$, $0.97(\mathrm{~d}, \mathrm{~J}=6.8 \mathrm{~Hz}, 3 \mathrm{H}), 1.93-2.00(\mathrm{~m}, 1 \mathrm{H}), 2.62(\mathrm{dd}, \mathrm{J}=3.6$ and $1.6 \mathrm{~Hz}, 1 \mathrm{H}), 3.58(\mathrm{br}$ s, $1 \mathrm{H}), 3.69(\mathrm{~s}, 3 \mathrm{H}), 3.74(\mathrm{~s}, 3 \mathrm{H}), 3.78(\mathrm{~s}, 3 \mathrm{H}), 5.26(\mathrm{~d}, \mathrm{~J}=1.6 \mathrm{~Hz}, 1 \mathrm{H}), 5.61(\mathrm{br} \mathrm{s}, 1 \mathrm{H})$, $6.81(\mathrm{~d}, \mathrm{~J}=8.4 \mathrm{~Hz}, 2 \mathrm{H}), 7.10(\mathrm{~d}, \mathrm{~J}=8.4 \mathrm{~Hz}, 2 \mathrm{H}) ;{ }^{13} \mathrm{C}-\mathrm{NMR}\left(\mathrm{CDCl}_{3}\right): 16.9\left(\mathrm{CH}_{3}\right), 20.3$ $\left(\mathrm{CH}_{3}\right), 33.1(\mathrm{CH}), 46.1(\mathrm{CH}), 51.5\left(\mathrm{CH}_{3}\right), 55.3\left(\mathrm{CH}_{3}\right), 57.2\left(\mathrm{CH}_{3}\right), 57.4(\mathrm{CH}), 110.1$ (CH), $113.9(\mathrm{CH}), 114.8(\mathrm{CH}), 128.3(\mathrm{CH}), 137.7(\mathrm{C}), 152.2(\mathrm{C}), 158.0(\mathrm{C}), 158.2(\mathrm{C})$, 167.6 (C). Anal. Calcd for $\mathrm{C}_{19} \mathrm{H}_{24} \mathrm{O}_{4}$ : C, 72.13; H, 7.65; Found: C, 72.22; H, 7.63. Compounds 9f and 10f. Spectroscopic data of 9f and 10f were obtained from a 1:1 mixture. ${ }^{1} \mathrm{H}-\mathrm{NMR}\left(\mathrm{CDCl}_{3}\right): 0.74(\mathrm{~d}, \mathrm{~J}=6.7 \mathrm{~Hz}, 3 \mathrm{H}), 0.86(\mathrm{~d}, \mathrm{~J}=6.7 \mathrm{~Hz}, 3 \mathrm{H}), 1.03(\mathrm{~d}, \mathrm{~J}$ $=6.9 \mathrm{~Hz}, 3 \mathrm{H}), 1.09(\mathrm{~d}, \mathrm{~J}=6.9 \mathrm{~Hz}, 3 \mathrm{H}), 1.96-2.01(\mathrm{~m}, 1 \mathrm{H}), 2.35-2.41(\mathrm{~m}, 1 \mathrm{H}), 2.64(\mathrm{~d}, \mathrm{~J}$ $=3.9 \mathrm{~Hz}, 1 \mathrm{H}), 3.39(\mathrm{br} \mathrm{s}, 1 \mathrm{H}), 3.60(\mathrm{~d}, \mathrm{~J}=2.7 \mathrm{~Hz}, 1 \mathrm{H}), 3.66(\mathrm{~d}, \mathrm{~J}=2.9 \mathrm{~Hz}, 1 \mathrm{H}), 3.68(\mathrm{~s}$, 3H), 3.74 (s, 3H), 3.77 (s, 3H), 3.78 (s, 3H), 5.39 (d, J = 2.9 Hz, 1H), 5.62 (br s, 2H), 
$5.98(\mathrm{~d}, \mathrm{~J}=1.7 \mathrm{~Hz}, 1 \mathrm{H}), 6.79(\mathrm{~d}, \mathrm{~J}=8.3 \mathrm{~Hz}, 2 \mathrm{H}), 6.82(\mathrm{~d}, \mathrm{~J}=8.4 \mathrm{~Hz}, 2 \mathrm{H}), 7.04(\mathrm{~d}, \mathrm{~J}=$ 8.4 Hz, 2H), $7.07(\mathrm{~d}, \mathrm{~J}=8.3,2 \mathrm{H}) ;{ }^{13} \mathrm{C}-\mathrm{NMR}\left(\mathrm{CDCl}_{3}\right): 16.1,16.3,20.6,21.5,31.5,33.7$, $45.6,46.1,51.0,52.3,55.2,55.3,56.9,59.2,107.5,107.8,113.9,115.1,124.5,128.1$, $128.3,136.8,137.4,155.4,158.1,158.2,158.4,162.9,166.5,167.3,170.6$.

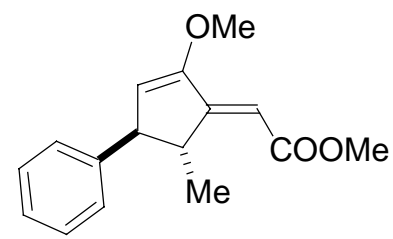

$9 g$

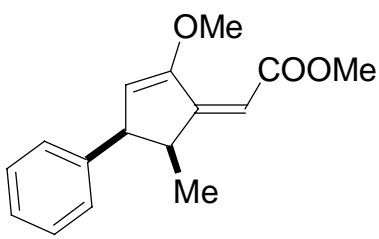

$10 \mathrm{~g}$

Compounds $9 \mathrm{~g}$ and 10g. The general procedure was followed using complex 1a (184 $\mathrm{mg}, 0.5 \mathrm{mmol})$, allene $3 \mathrm{~h}(168 \mathrm{mg}, 1.5 \mathrm{mmol})$ and $[\mathrm{Rh}$ (naphthalene)(cod)][SbF 6$]$ (29 $\mathrm{mg}, 0.05 \mathrm{mmol})$ in $\mathrm{CH}_{2} \mathrm{Cl}_{2}(10 \mathrm{~mL})$. Final chromatographic purification using a 5:1 mixture of hexane:ethyl acetate as eluent afforded an unseparable mixture of $\mathbf{9 g}$ and $\mathbf{1 0 g}$ (86 $\mathrm{mg}, 60 \%$ ). Spectroscopic and analytical data of $\mathbf{9 g}$ and $\mathbf{1 0 g}$ were obtained from this mixture. Compound 9g: ${ }^{1} \mathrm{H}-\mathrm{NMR}\left(\mathrm{CDCl}_{3}\right)$ : $1.36(\mathrm{~d}, \mathrm{~J}=7.1 \mathrm{~Hz}, 3 \mathrm{H}) ; 3.40-3.44(\mathrm{~m}$, 1H), 3.50 (br s, 1H), $3.70(\mathrm{~s}, 3 \mathrm{H}), 3.78(\mathrm{~s}, 3 \mathrm{H}), 5.42(\mathrm{~d}, \mathrm{~J}=2.9 \mathrm{~Hz}, 1 \mathrm{H}), 5.97$ (d, J = 1.5 $\mathrm{Hz}, 1 \mathrm{H}), 7.13-7.32(\mathrm{~m}, 5 \mathrm{H}) ;{ }^{13} \mathrm{C}-\mathrm{NMR}\left(\mathrm{CDCl}_{3}\right): 21.7\left(\mathrm{CH}_{3}\right), 44.9(\mathrm{CH}), 51.0(\mathrm{CH}), 55.1$ $\left(\mathrm{CH}_{3}\right), 56.9\left(\mathrm{CH}_{3}\right), 107.6(\mathrm{CH}), 113.4(\mathrm{CH}), 126.5(\mathrm{CH}), 126.7(\mathrm{CH}), 127.1(\mathrm{CH}), 144.7$ (C), $157.2(\mathrm{C}), 163.6(\mathrm{C}), 167.2(\mathrm{C})$. Compound 10g: ${ }^{1} \mathrm{H}-\mathrm{NMR}\left(\mathrm{CDCl}_{3}\right): 1.30(\mathrm{~d}, \mathrm{~J}=$ $7.1 \mathrm{~Hz}, 3 \mathrm{H}), 2.63-2.68(\mathrm{~m}, 1 \mathrm{H}), 3.47(\mathrm{br} \mathrm{s}, 1 \mathrm{H}), 3.72(\mathrm{~s}, 3 \mathrm{H}), 3.82(\mathrm{~s}, 3 \mathrm{H}), 5.06$ (br s, $1 \mathrm{H}), 5.67(\mathrm{~m}, 1 \mathrm{H})$, otherwise as for the major isomer; ${ }^{13} \mathrm{C}-\mathrm{NMR}\left(\mathrm{CDCl}_{3}\right): 19.4\left(\mathrm{CH}_{3}\right)$, $49.9(\mathrm{CH}), 52.3(\mathrm{CH}), 55.1\left(\mathrm{CH}_{3}\right), 54.2\left(\mathrm{CH}_{3}\right), 107.1(\mathrm{CH}), 122.3(\mathrm{CH}), 126.8(\mathrm{CH})$, $128.5(\mathrm{CH}), 128.6(\mathrm{CH}), 144.0(\mathrm{C}), 155.0(\mathrm{C}), 167.5(\mathrm{C}), 170.7(\mathrm{C})$. Anal. Calcd for $\mathrm{C}_{16} \mathrm{H}_{18} \mathrm{O}_{3}:$ C, 74.39; H, 7.02; Found: C, 74.53; H, 7.09. 


\section{Removal of the Tosyl Group in Compound 4j with Li/Naphthalene}

To a suspension of lithium powder $(21 \mathrm{mg}, 3.0 \mathrm{mmol})$, and naphthalene $(2 \mathrm{mg}, 0.016$ mmol) in THF ( $5 \mathrm{~mL}$ ) was slowly added a solution of compound $\mathbf{4 j}$ ( $90 \mathrm{mg}, 0.21 \mathrm{mmol}$ ) at $-78{ }^{\circ} \mathrm{C}$. Stirring was continued for $6 \mathrm{~h}$. The resulting mixture was then hydrolysed with water $(5 \mathrm{~mL})$ and extracted with diethyl ether $(2 \times 20 \mathrm{~mL})$. The organic layer was dried over anhydrous $\mathrm{Na}_{2} \mathrm{SO}_{4}$. Solvents were evaporated in vacuo and the resulting residue was purified by flash chromatography $\left(\mathrm{SiO}_{2}\right.$, hexane:ethyl acetate 5:1) affording compound 5 (32 mg, 57\%).

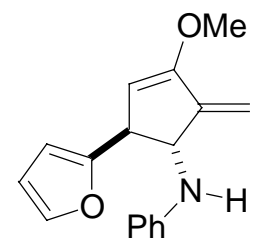

5

${ }^{1} \mathrm{H}-\mathrm{NMR}\left(\mathrm{CDCl}_{3}\right): 3.79(\mathrm{~s}, 3 \mathrm{H}), 3.91(\mathrm{t}, \mathrm{J}=2.6 \mathrm{~Hz}, 1 \mathrm{H}), 4.04$ (br signal, $\left.1 \mathrm{H}\right), 4.51(\mathrm{~d}, J$ $=2.0 \mathrm{~Hz}, 1 \mathrm{H}), 5.03$ (br s, 1H), 5.11 (br s, 1H), $5.32(\mathrm{~d}, J=2 \mathrm{~Hz}, 1 \mathrm{H}), 6.10(\mathrm{~d}, \mathrm{~J}=3.2$ $\mathrm{Hz}, 1 \mathrm{H}), 6.32-6.34(\mathrm{~m}, 1 \mathrm{H}), 6.60(\mathrm{~d}, J=7.6 \mathrm{~Hz}, 2 \mathrm{H}), 6.70-6.74(\mathrm{~m}, 1 \mathrm{H}), 7.16(\mathrm{~d}, J=7.2$ $\mathrm{Hz}, 2 \mathrm{H}), 7.39$ (br s, 1H); ${ }^{13} \mathrm{C}-\mathrm{NMR}\left(\mathrm{CDCl}_{3}\right): 46.9(\mathrm{CH}), 56.5\left(\mathrm{CH}_{3}\right), 60.4(\mathrm{CH}), 103.0$ $(\mathrm{CH}), 105.0\left(\mathrm{CH}_{2}\right), 105.3(\mathrm{CH}), 110.3(\mathrm{CH}), 113.3(\mathrm{CH}), 117.6(\mathrm{CH}), 129.2(\mathrm{CH})$, $141.5(\mathrm{CH}), 146.8(\mathrm{C}), 147.9(\mathrm{C}), 156.9(\mathrm{C}), 157.5(\mathrm{C})$. Anal. Calcd for $\mathrm{C}_{17} \mathrm{H}_{17} \mathrm{NO}_{2}$ : C, 76.38; H, 6.41; N, 5.24; Found: C, 76.27; H, 6.54; N, 5.22.

\section{General Procedure for the Synthesis of 2-Methylenecyclopentanones 11}

$2 \mathrm{M} \mathrm{HCl}(1 \mathrm{~mL})$ was added to a solution of the corresponding compound $(0.1 \mathrm{mmol})$ in THF ( $5 \mathrm{~mL})$. The mixture was stirred for $2 \mathrm{~h}$ at $\mathrm{rt}$. Then water $(5 \mathrm{~mL})$ was added an the mixture extracted with diethyl ether $(3 \times 10 \mathrm{~mL})$. The combined organic layers were 
dried $\left(\mathrm{Na}_{2} \mathrm{SO}_{4}\right)$. The solvent was removed in vacuo and the resulting residue was purified by flash chromatography $\left(\mathrm{SiO}_{2}\right.$, mixtures of hexane:ethyl acetate).

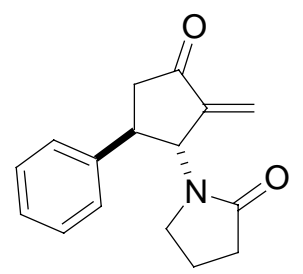

11a

Compound 11a. The general procedure was followed using compound $\mathbf{4 b}$ ( $27 \mathrm{mg}, 0.1$ mmol) in THF (5 mL). Final chromatographic purification using a 1:1 mixture of hexane:ethyl acetate as eluent afforded 11a $(25 \mathrm{mg}, 98 \%) .{ }^{1} \mathrm{H}-\mathrm{NMR}\left(\mathrm{CDCl}_{3}\right): 2.00$ $2.07(\mathrm{~m}, 2 \mathrm{H}), 2.28-2.46(\mathrm{~m}, 2 \mathrm{H}), 2.53(\mathrm{dd}, \mathrm{J}=18.0$ and $12.7 \mathrm{~Hz}, 1 \mathrm{H}), 2.87(\mathrm{dd}, \mathrm{J}=18.0$ and $7.5 \mathrm{~Hz}, 1 \mathrm{H}), 3.29-3.44(\mathrm{~m}, 3 \mathrm{H}), 5.22(\mathrm{~d}, \mathrm{~J}=2.7 \mathrm{~Hz}, 1 \mathrm{H}), 5.50-5.55(\mathrm{~m}, 1 \mathrm{H}), 6.23$ $(\mathrm{d}, \mathrm{J}=3.3 \mathrm{~Hz}, 1 \mathrm{H}), 7.26-7.35(\mathrm{~m}, 5 \mathrm{H}) ;{ }^{13} \mathrm{C}-\mathrm{NMR}\left(\mathrm{CDCl}_{3}\right): 18.4\left(\mathrm{CH}_{2}\right), 30.9\left(\mathrm{CH}_{2}\right), 42.4$ $\left(\mathrm{CH}_{2}\right), 42.8(\mathrm{CH}), 45.7\left(\mathrm{CH}_{2}\right), 57.9(\mathrm{CH}), 118.7\left(\mathrm{CH}_{2}\right), 127.0(\mathrm{CH}), 127.5(\mathrm{CH}), 129.0$ $(\mathrm{CH}), 138.8(\mathrm{C}), 142.5(\mathrm{C}), 175.6(\mathrm{C}), 201.2(\mathrm{C}) . \mathrm{IR}\left(\mathrm{CH}_{2} \mathrm{Cl}_{2}\right)$ : v 1732, 1682, $1634 \mathrm{~cm}^{-1}$. Anal. Calcd for $\mathrm{C}_{16} \mathrm{H}_{17} \mathrm{NO}_{2}$ : C, 75.27; H, 6.71; Found: C, 75.32; H, 6.86.

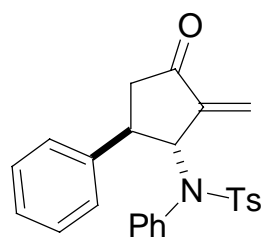

$11 b$

Compound 11b. The general procedure was followed using compound $\mathbf{4 h}$ (43 $\mathrm{mg}, 0.1$ $\mathrm{mmol})$ in THF (5 mL). Final chromatographic purification using a 3:1 mixture of hexane:ethyl acetate as eluent afforded 11b (39 mg, $95 \%) .{ }^{1} \mathrm{H}-\mathrm{NMR}\left(\mathrm{CDCl}_{3}\right): 2.37$ (s, 3H), $2.41(\mathrm{dd}, J=18.2$ and $11.6 \mathrm{~Hz}, 1 \mathrm{H}), 2.62(\mathrm{dd}, J=18.2$ and $8.3 \mathrm{~Hz}, 1 \mathrm{H}), 3.02-3.10$ $(\mathrm{m}, 1 \mathrm{H}), 5.80-5.84(\mathrm{~m}, 1 \mathrm{H}), 6.01(\mathrm{~d}, J=2.7 \mathrm{~Hz}, 1 \mathrm{H}), 6.50(\mathrm{~d}, J=3.1 \mathrm{~Hz}, 1 \mathrm{H}), 6.91(\mathrm{~d}, J$ $=8.0 \mathrm{~Hz}, 2 \mathrm{H}), 7.02(\mathrm{~d}, J=8.0 \mathrm{~Hz}, 2 \mathrm{H}), 7.21-7.27(\mathrm{~m}, 10 \mathrm{H}) ;{ }^{13} \mathrm{C}-\mathrm{NMR}\left(\mathrm{CDCl}_{3}\right): 21.4$ 
$\left(\mathrm{CH}_{3}\right), 43.7(\mathrm{CH}), 46.4\left(\mathrm{CH}_{2}\right), 66.3(\mathrm{CH}), 122.8\left(\mathrm{CH}_{2}\right), 127.2(\mathrm{CH}), 127.4(\mathrm{CH}), 127.5$ (CH), $127.7(\mathrm{CH}), 129.1(\mathrm{CH}), 129.2(\mathrm{CH}), 129.3(\mathrm{CH}), 132.6(\mathrm{CH}), 135.5(\mathrm{C}), 137.1$ (C), 140.1 (C), 143.2 (C), 144.3 (C), 201.3 (C). Anal. Calcd for $\mathrm{C}_{25} \mathrm{H}_{23} \mathrm{NO}_{3} \mathrm{~S}$ : C, 71.92; H, 5.55; N, 3.35; Found: C, 71.80; H, 5.59; N, 3.38.

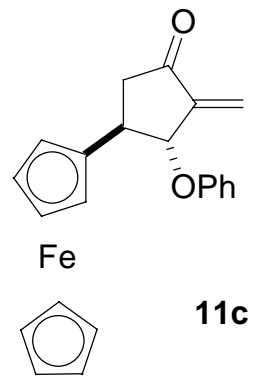

Compound 11c. The general procedure was followed using compound 6e (39 mg, 0.1 $\mathrm{mmol})$ in THF (5 mL). Final chromatographic purification using a 5:1 mixture of hexane:ethyl acetate as eluent afforded 11c $(29 \mathrm{mg}, 77 \%) .{ }^{1} \mathrm{H}-\mathrm{NMR}\left(\mathrm{CDCl}_{3}\right): 2.69$ (dd, $\mathrm{J}=18.4$ and $7.8 \mathrm{~Hz}, 1 \mathrm{H}), 3.05(\mathrm{dd}, J=18.4$ and $8.6 \mathrm{~Hz}, 1 \mathrm{H}), 3.33-3.44(\mathrm{~m}, 1 \mathrm{H}), 4.01-$ $4.02(\mathrm{~m}, 1 \mathrm{H}), 4.03-2.19(\mathrm{~s}+\mathrm{m}, 8 \mathrm{H}), 5.13-5.16(\mathrm{~m}, 1 \mathrm{H}), 5.58(\mathrm{~d}, J=2.9 \mathrm{~Hz}, 1 \mathrm{H}), 6.25(\mathrm{~d}$, $J=2.9 \mathrm{~Hz}, 1 \mathrm{H}), 6.96-7.07(\mathrm{~m}, 3 \mathrm{H}), 7.29-7.35(\mathrm{~m}, 2 \mathrm{H}) ;{ }^{13} \mathrm{C}-\mathrm{NMR}\left(\mathrm{CDCl}_{3}\right): 39.6(\mathrm{CH})$, $43.0\left(\mathrm{CH}_{2}\right), 66.1(\mathrm{CH}), 67.8(\mathrm{CH}), 67.9(\mathrm{CH}), 68.0(\mathrm{CH}), 68.5(\mathrm{CH}), 83.1(\mathrm{CH}), 88.9$ (C), $115.8(\mathrm{CH}), 121.6(\mathrm{CH}), 122.3\left(\mathrm{CH}_{2}\right), 129.7(\mathrm{CH}), 144.9(\mathrm{C}), 157.8(\mathrm{C}), 202.9(\mathrm{C})$. IR $\left(\mathrm{CH}_{2} \mathrm{Cl}_{2}\right): v 1727,1651,1597,1493,1381,1228,1097 \mathrm{~cm}^{-1}$. Anal. Calcd for $\mathrm{C}_{22} \mathrm{H}_{20} \mathrm{FeO}_{2}$ : C, 70.99; H, 5.42; Found: C, 71.16; H, 5.35.

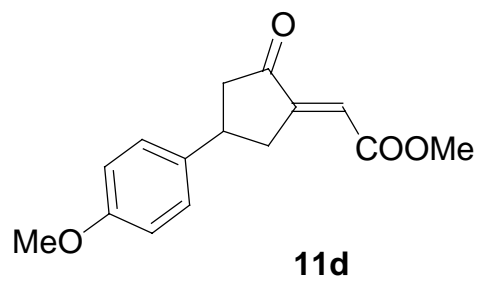

Compound 11d. The general procedure was followed using compound 7a (27 mg, 0.1 $\mathrm{mmol})$ in THF (5 mL). Final chromatographic purification using a 1:1 mixture of hexane:ethyl acetate as eluent afforded 11d $(23 \mathrm{mg}, 89 \%) .{ }^{1} \mathrm{H}-\mathrm{NMR}\left(\mathrm{CDCl}_{3}\right): 2.52$ (dd, 
$\mathrm{J}=18.1$ and $10.0 \mathrm{~Hz}, 1 \mathrm{H}), 2.80-3.01(\mathrm{~m}, 2 \mathrm{H}), 3.39-3.45(\mathrm{~m}, 1 \mathrm{H}), 3.66-3.71(\mathrm{~m}, 1 \mathrm{H})$, $3.78(\mathrm{~s}, 3 \mathrm{H}), 3.80(\mathrm{~s}, 3 \mathrm{H}), 6.58(\mathrm{t}, \mathrm{J}=2.9,1 \mathrm{H}), 6.88(\mathrm{~d}, \mathrm{~J}=8.7 \mathrm{~Hz}, 2 \mathrm{H}), 7.15(\mathrm{~d}, \mathrm{~J}=8.7$ $\mathrm{Hz}, 2 \mathrm{H}) ;{ }^{13} \mathrm{C}-\mathrm{NMR}\left(\mathrm{CDCl}_{3}\right): 37.8\left(\mathrm{CH}_{2}\right), 37.9(\mathrm{CH}), 45.8\left(\mathrm{CH}_{2}\right), 51.9\left(\mathrm{CH}_{3}\right), 55.3\left(\mathrm{CH}_{3}\right)$, $114.2(\mathrm{CH}), 119.5(\mathrm{CH}), 127.6(\mathrm{CH}), 134.8(\mathrm{C}), 150.9(\mathrm{C}), 158.5(\mathrm{C}), 166.0(\mathrm{C}), 205.8$ (C). IR $\left(\mathrm{CH}_{2} \mathrm{Cl}_{2}\right): v 1714,1514,1220 \mathrm{~cm}^{-1}$. Anal. Calcd for $\mathrm{C}_{15} \mathrm{H}_{16} \mathrm{O}_{4}: \mathrm{C}, 69.22 ; \mathrm{H}$, 6.20; Found: C, 69.35; H, 6.14.

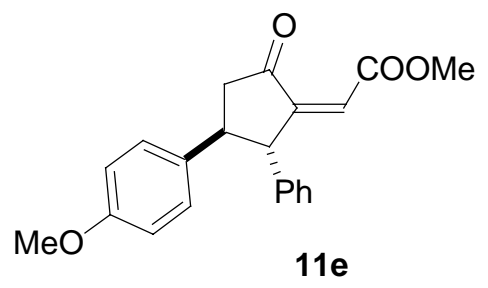

Compound 11e. The general procedure was followed using compound 8a (35 mg, 0.1 mmol) in THF $(5 \mathrm{~mL})$. Final chromatographic purification using a 1:1 mixture of hexane:ethyl acetate as eluent afforded 11e $(18 \mathrm{mg}, 53 \%) .{ }^{1} \mathrm{H}-\mathrm{NMR}\left(\mathrm{CDCl}_{3}\right): 2.68(\mathrm{dd}$, $\mathrm{J}=18.6$ and $6.6 \mathrm{~Hz}, 1 \mathrm{H}), 2.96(\mathrm{dd}, \mathrm{J}=18.6$ and $8.3 \mathrm{~Hz}, 1 \mathrm{H}), 3.41-3.47(\mathrm{~s}+\mathrm{m}, 4 \mathrm{H}), 3.80$ (s, 3H), $4.60(\mathrm{dd}, \mathrm{J}=5.2$ and $2.9 \mathrm{~Hz}, 1 \mathrm{H}), 6.83-6.86(\mathrm{~m}, 3 \mathrm{H}), 7.01-7.06(\mathrm{~m}, 4 \mathrm{H}), 7.20$ $7.23(\mathrm{~m}, 1 \mathrm{H}), 7.26-7.30(\mathrm{~m}, 2 \mathrm{H}) ;{ }^{13} \mathrm{C}-\mathrm{NMR}\left(\mathrm{CDCl}_{3}\right): 44.1\left(\mathrm{CH}_{2}\right), 48.1(\mathrm{CH}), 51.6$ $\left(\mathrm{CH}_{3}\right), 55.1(\mathrm{CH}), 55.2\left(\mathrm{CH}_{3}\right), 114.2(\mathrm{CH}), 122.9(\mathrm{CH}), 126.6(\mathrm{CH}), 127.1(\mathrm{CH}), 127.8$ $(\mathrm{CH}), 128.6(\mathrm{CH}), 134.8(\mathrm{C}), 142.6(\mathrm{C}), 151.3(\mathrm{C}), 158.5(\mathrm{C}), 165.7(\mathrm{C}), 206.0(\mathrm{C})$. Anal. Calcd for $\mathrm{C}_{21} \mathrm{H}_{20} \mathrm{O}_{4}$ : C, 74.98; H, 5.99; Found: C, 75.07; H, 6.12. 